\title{
Unified mechanism of local drivers in a percolation model of atrial fibrillation
}

\author{
Max Falkenberg $\odot,{ }^{1,2,3}$ Andrew J. Ford, ${ }^{1}$ Anthony C. Li, ${ }^{1}$ Robert Lawrence, ${ }^{1}$ Alberto Ciacci, ${ }^{1,2,3}$ \\ Nicholas S. Peters $\mathbb{C}^{3,4, *}$ and Kim Christensen (1) ${ }^{1,2,3, \dagger}$ \\ ${ }^{1}$ Blackett Laboratory, Imperial College London, London SW7 2AZ, United Kingdom \\ ${ }^{2}$ Centre for Complexity Science, Imperial College London, London SW7 2AZ, United Kingdom \\ ${ }^{3}$ Centre for Cardiac Engineering, Imperial College London, London W12 ONN, United Kingdom \\ ${ }^{4}$ National Heart \& Lung Institute, Imperial College London, London, W12 ONN, United Kingdom
}

(Received 29 October 2018; revised manuscript received 28 May 2019; published 9 December 2019)

\begin{abstract}
The mechanisms of atrial fibrillation (AF) are poorly understood, resulting in disappointing success rates of ablative treatment. Different mechanisms defined largely by different atrial activation patterns have been proposed and, arguably, this dispute has slowed the progress of AF research. Recent clinical evidence suggests a unifying mechanism of local drivers based on sustained reentrant circuits in the complex atrial architecture. Here, we present a percolation inspired computational model showing spontaneous emergence of AF that strongly supports, and gives a theoretical explanation for, the clinically observed diversity of activation. We show that the difference in surface activation patterns is a direct consequence of the thickness of the discrete network of heart muscle cells through which electrical signals percolate to reach the imaged surface. The model naturally follows the clinical spectrum of AF spanning sinus rhythm, paroxysmal AF, and persistent AF as the decoupling of myocardial cells results in the lattice approaching the percolation threshold. This allows the model to make the prediction that, for paroxysmal AF, reentrant circuits emerge near the endocardium, but in persistent $\mathrm{AF}$ they emerge deeper in the bulk of the atrial wall. If experimentally verified, this may go towards explaining the lowering ablation success rate as AF becomes more persistent.
\end{abstract}

DOI: 10.1103/PhysRevE.100.062406

Atrial fibrillation (AF) is the most common cardiac arrhythmia [1], but our understanding of the underlying mechanisms is still poor [2-9]. There is growing evidence in favor of local drivers as a sustaining mechanism of AF [4,9-18]. However, there is debate about the mechanistic origin of local drivers: Some studies have identified the drivers as rotors (reentrant drivers) [12,13], whereas others have identified the drivers as focal points [11].

Recently, a study with "the potential to unify some of the previous discrepant observations" [19] has suggested that both focal and reentrant drivers may be explained by the presence of small reentrant circuits [14,20]. Using highresolution simultaneous optical mapping of the endocardium (inner heart wall) and epicardium (outer heart wall) in explanted, diseased human hearts, the study shows that stable transmural reentrant circuits may project differently onto the endo- and epicardium. Projections typically appear as rotational activity on the endocardium and focal (breakthrough) points on the epicardium. Hence, the apparently incompatible two-dimensional (2D) projections onto the atrial walls are consistent with a single underlying mechanism of localized reentrant circuits in the transmural region [14-16].

\footnotetext{
*Clinical Corresponding author: n.peters@imperial.ac.uk

†Physics Corresponding author: k.christensen@imperial.ac.uk
}

Published by the American Physical Society under the terms of the Creative Commons Attribution 4.0 International license. Further distribution of this work must maintain attribution to the author(s) and the published article's title, journal citation, and DOI.
Leading clinicians have called for further work "to confirm and extend these observations" [19]. In this paper, we develop a percolation based model of AF initiation in pursuit of this goal. Our aim is to understand the origin of the $\mathrm{AF}$ mechanism, how it changes as $\mathrm{AF}$ becomes more or less persistent, and how this effects the success rate of ablation. We take a physics approach to modeling, focusing on the essential features of the phenomenon as opposed to the fine details. This is much simpler than more biophysically realistic models and allows for concrete predictions based on large scale statistics not accessible in a laboratory setting.

The model generates activation wavefronts that propagate in a three-dimensional (3D) medium mimicking the complex discrete fiber structure of the atria. AF emerges spontaneously via the formation of spatially stable but temporally intermittent 3D reentrant circuits when the fiber network decouples, e.g., through fibrosis or fatty infiltration [21]. The model predicts that these circuits should have a minimum length of $12.5 \mathrm{~mm}$, comparable with the $15 \mathrm{~mm}$ quoted in Ref. [14]. Reentrant circuits are quasi-one-dimensional with isolated fibers that have a width of around $2 \mathrm{~mm}$, making their identification with clinically available mapping technologies difficult [22].

Our results demonstrate how 3D reentrant circuits emerge and how they are distributed in the heart wall when the coupling between cells is lowered, closely matching key clinical observations in Ref. [14]. The observed activation patterns fundamentally change as a function of the depth from the imaged surface to the driving reentrant circuit. If this depth is large, we observe focal (breakthrough) activity. If this depth is small, we observe reentrant or rotational activity. 
The model predicts that the drivers of paroxysmal AF are located near the endocardium whereas the drivers of persistent $\mathrm{AF}$ are uniformly distributed through the atrial wall. Consistent with clinical experience, the former are more easily ablated than the latter. We test this by numerically simulating focal ablation. We find that ablation lesions that do not penetrate the full depth into the tissue are significantly less likely to destroy a reentrant circuit in persistent $\mathrm{AF}$ than in paroxysmal AF. Additionally, we show that in persistent $\mathrm{AF}$, even if ablation successfully destroys a local source, the existence of multiple coexisting circuits prevents the global termination of $\mathrm{AF}$.

\section{MODEL}

Electrical signals in the heart are mediated by discrete heart cells arranged in long, intertwined fibers. This motivates the construction of a 3D lattice model where each node acts as a single cell (or block of cells), and nodes are connected stochastically to their neighbors to mimic the branching structure of discrete heart muscle fibers, generalizing a 2D model [23]. Our model simulates the initiation and maintenance of AF from local microanatomical reentrant circuits. We do not investigate local drivers linked to cardiomyocyte automaticity, or the maintenance of fibrillation through the formation of rotors or scroll waves. Such phenomena are best studied using continuous reaction-diffusion models of cardiac electrophysiology [24].

Discrete models of cardiac tissue are well established historically and have recently regained popularity $[23,25-32]$. In these models, the onset of reentry has been associated with the approach from above of the bond occupation probability to the percolation threshold [29]. Some models have been extended to three dimensions to highlight the importance of the thickness of the atrial wall to the probability of reentry [30]. Specifically when investigating the role of fibrosis in cardiac arrhythmia, which is the approach taken here, there have been suggestions that discrete models are preferable over continuous models [33]. Note, mechanistically it is important to distinguish between $3 \mathrm{D}$ vortices in a continuous substrate which exhibit functional reentry and 3D reentrant circuits where electrically isolated fibers exhibit structural reentry. The clinical results in Ref. [14] concern structural reentrant circuits and not the wider literature on rotors and scroll waves [24,34].

We consider a simplified $L_{x} \times L_{y} \times L_{z}$ pipe topology of the atria with open boundaries in $x$ and $z$, and periodic boundaries in $y$. Nodes are connected longitudinally to their neighbors in the $x$ direction with spatial frequency $v_{\|}$and transversely, in the $y$ and $z$ directions, with frequency $v_{\perp}$. Once the network is defined at the start of a simulation, it is fixed for the remainder of the simulation.

Each node takes one of three states: resting, where the node can be excited by an active neighbor; excited; or refractory, where for $\tau$ time steps after excitation a node cannot be reexcited. We define the sinus node as nodes at the boundary, $x=0$, which excites every $T$ time steps. Model parameters $L_{x}=L_{y}=200, L_{z}=25, T=220$, and $\tau=50$ are informed by clinically observed values (see Appendix A). The model results are robust against changes in these parameters. A small fraction of nodes, $\delta$, are susceptible to conduction block. With probability $\epsilon$, these nodes fail to activate when their neighbors excite. When the sinus node excites in normal conduction, wavefronts are initiated at $x=0$ and propagate smoothly in the $+x$ direction and terminate at $x=L_{x}$. However, reentrant circuits can form when nodes are sufficiently decoupled, through fibrosis $[29,35]$, or otherwise, such that the shortest closed loop from a node back to itself is partially isolated from the remaining tissue, and the path length, in units of propagation time, exceeds the refractory period $\tau$. The formation of these reentrant circuits drives fibrillatory activity [23,36] (see Fig. 1). Further examples are shown in Appendix D including figures showing close-up examples of reentrant circuits and focal breakthrough points. Videos of typical activation patterns are included in the Supplemental Material [37] and are captioned in Appendix E.

Reference [14] finds that reentry activity is typically observed on the endocardial surface, whereas focal points are often found on the epicardial surface. Additionally, there is much stronger longitudinal coupling on the endo- than the epicardial surface. To test whether fiber orientation can account for the distribution of activation patterns observed clinically, we consider a homogeneous and inhomogeneous model. In the homogeneous model all nodes are connected to their neighbors with the same frequencies, $v_{\|}$and $v_{\perp}$. In the inhomogeneous model, the variation in fiber direction changes with depth. Here we fix the average decoupling of nodes, $\bar{v}=\left(2 v_{\|}+4 v_{\perp}\right) / 6$, and vary linearly the average fiber angle, $\Delta \theta=\tan ^{-1}\left(v_{\perp} / v_{\|}\right)$, in each layer from $\Delta \theta_{\text {endo }}=24^{\circ}$ at the endocardium $(z=0)$ to $\Delta \theta_{\text {epi }}=42^{\circ}$ at the epicardium $(z=24)$. These values are taken from clinical data [16]. The introduction of fiber inhomogeneity into the model results in reentrant circuits preferentially anchoring to the endocardium. An example of this can be seen in Fig. 2, which shows a cross section of the model activation patterns from the endocardium to the epicardium. A reentrant circuit is shown anchored to the endocardium, with the activation wavefronts propagating from one end of the circuit to the epicardial surface and emerging as a focal breakthrough point.

To study the effect of local ablation in the model, we identify the first location at which a reentrant circuit forms. A new simulation with the identical structure is generated and the nodes at the identified location are destroyed, mimicking focal ablation, up to a prespecified depth (see Appendix C for details). This process is repeated until the locations of the first ten identified reentrant circuits are ablated. This allows us to study (a) the global success rate of ablation as a function of $\bar{v}$ by measuring the probability that ablation prevents $\mathrm{AF}$ emerging anywhere in the tissue and (b) the local success rate of ablation as a function of the ablation depth $z$, by measuring the probability that an ablation, that does not penetrate the full depth into the tissue, destroys the reentrant circuit at a given location.

\section{RESULTS}

The phase spaces for the risk of entering AF for the homogeneous and inhomogeneous models are shown in Fig. 3. For large values of the coupling parameters (no fibrosis) the model exhibits sinus rhythm indefinitely. As the coupling 


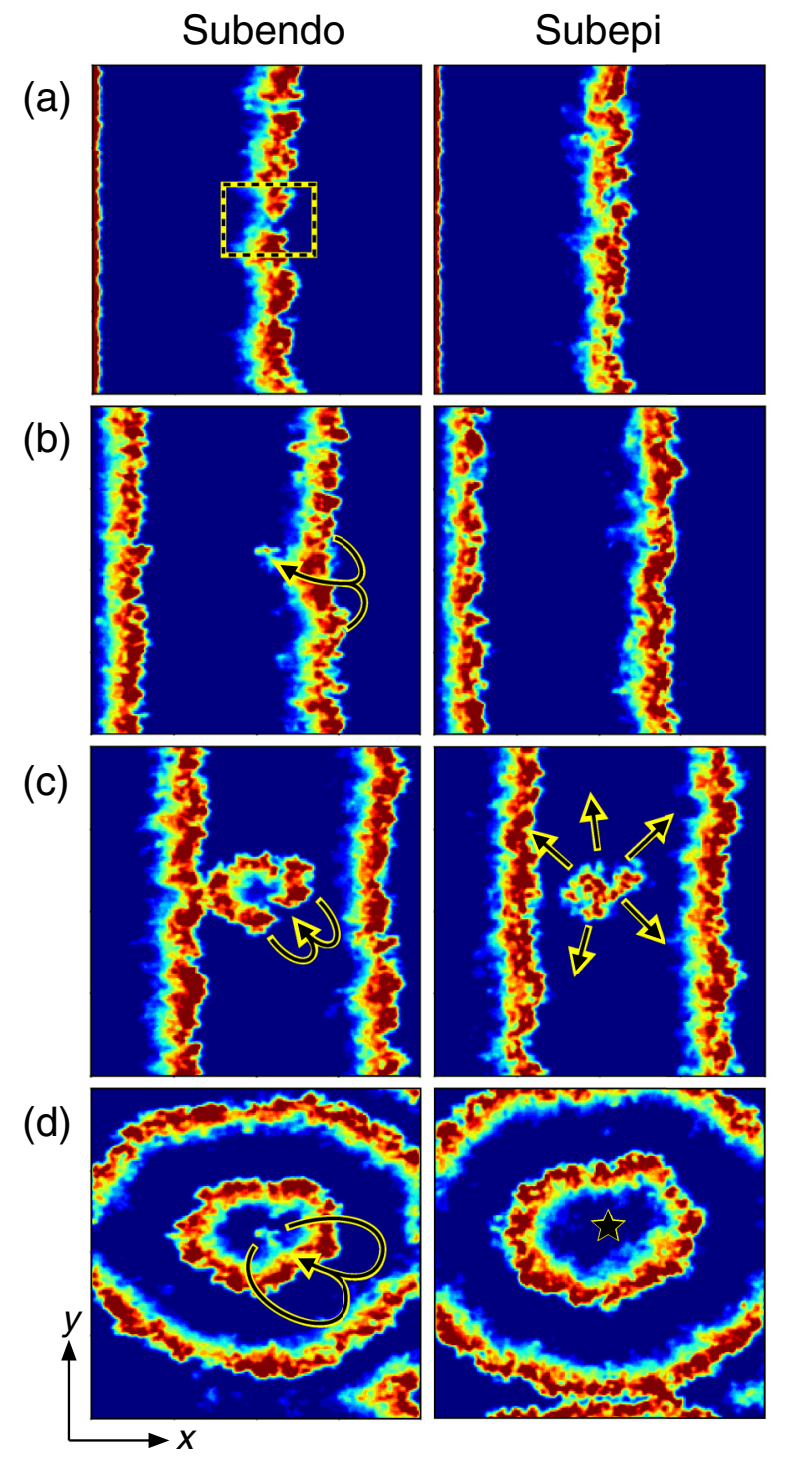

FIG. 1. The emergence of AF in the inhomogeneous model with simultaneous endo- $(z=0)$ and epicardial $(z=24)$ imaging. Smoothing has been applied for clarity. Red, excited nodes; blue, resting (excitable) nodes; other, refractory (unexcitable) nodes. (a) Planar wavefronts propagating during sinus rhythm. The dotted box indicates a gap in the wavefront formed by conduction block. (b) Endo view: The arrow indicates an excitation reentering the gap in the wavefront. Epi view: Reentry is not observed. (c) Emergence of fibrillatory activity. (d) Maintenance of fibrillatory activity. Epi view: Activity emerges on the surface as a point source (breakthrough activity) located at the star. See video A in Supplemental Material [37].

reduces, a small number of reentrant circuits can form. Here we observe paroxysmal AF with intermittent episodes of irregular activity. As the coupling is even further reduced, the model enters persistent AF where, once AF has been initiated, the model will never return to sinus rhythm without external intervention. These results are consistent with recent evidence showing that local drivers anchor at or near fibrotic lesions [13], with fibrosis increasing the number of reentrant regions and the time spent in AF [38]. (a)

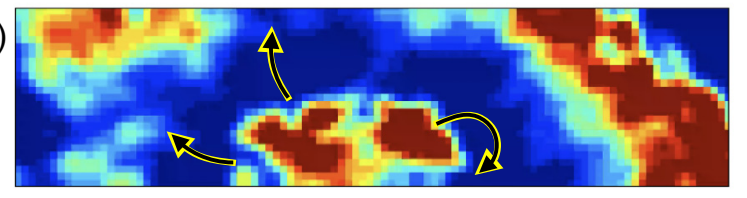

(b)

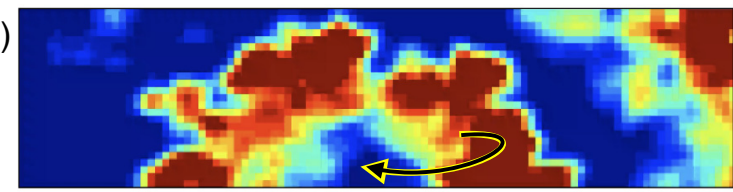

(c)
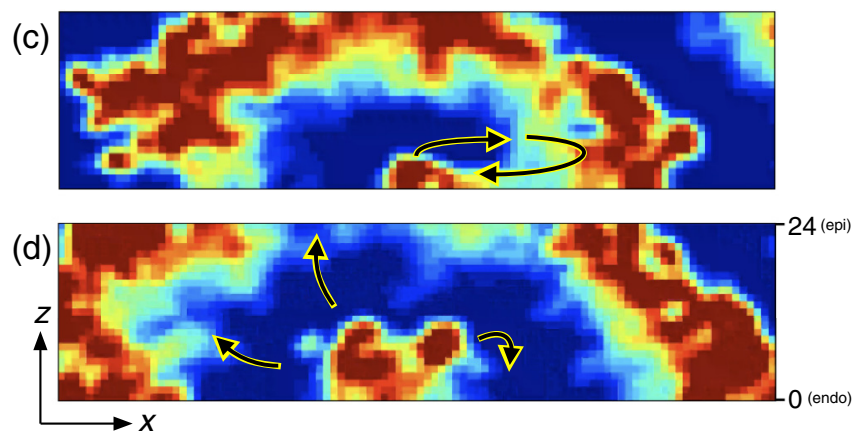

FIG. 2. A cross section of the model during AF showing a closeup of the $x-z$ plane (fixed $y$ coordinate). The cross section has been chosen to align with a reentrant circuit. Arrows indicate the movement of wavefronts. The top (bottom) surface of each panel corresponds to the epicardium (endocardium). A reentrant circuit can be seen near the endocardium with activity (a) moving around an isolated fiber, (b, c) reentering the isolated fiber, and (d) emitting fibrillatory waves from the left end of the isolated fiber. Fibrillatory activity spreads from the endocardium to the epicardium through the complex fiber structure of the model. This results in a wide variety of possible breakthrough patterns on the epicardial surface. When viewed from the endocardium, reentrant activity will be clearly visible since the isolated fiber lies along the endocardial surface. When viewed from the epicardial surface, the wavefronts propagating from the reentrant circuit may emerge as a single breakthrough point, or as multiple breakthrough points simultaneously. See video D in Supplemental Material [37]. Scale: $100 \times 25$ nodes.

In the homogeneous model the endo- and epicardium are equivalent, and we find that in paroxysmal AF the majority of drivers form equivalently on either surface, whereas for persistent AF the majority of drivers form in the bulk and not on the surfaces (see Fig. 16 in Appendix D).

The inhomogeneous model breaks the symmetry between the endo- and epicardium. Here we find that in paroxysmal AF drivers form preferentially near the endocardial surface, with very few drivers forming on the epicardial surface and almost none in the bulk of the atria [see Fig. 3(b), inset 1]. However, in persistent AF, drivers are uniformly distributed throughout the atrial wall [see Fig. 3(b), inset 2]. Hence, as AF becomes more persistent, the average position of drivers moves away from the endocardium and into the bulk of the atrial wall. The variation in fiber orientation is the only asymmetry between the endo- and epicardium and is therefore responsible for the asymmetry in the reentrant circuit depths. Because the method by which reentrant circuits form is fully local [39], the presence of an isolated fiber at one point in 

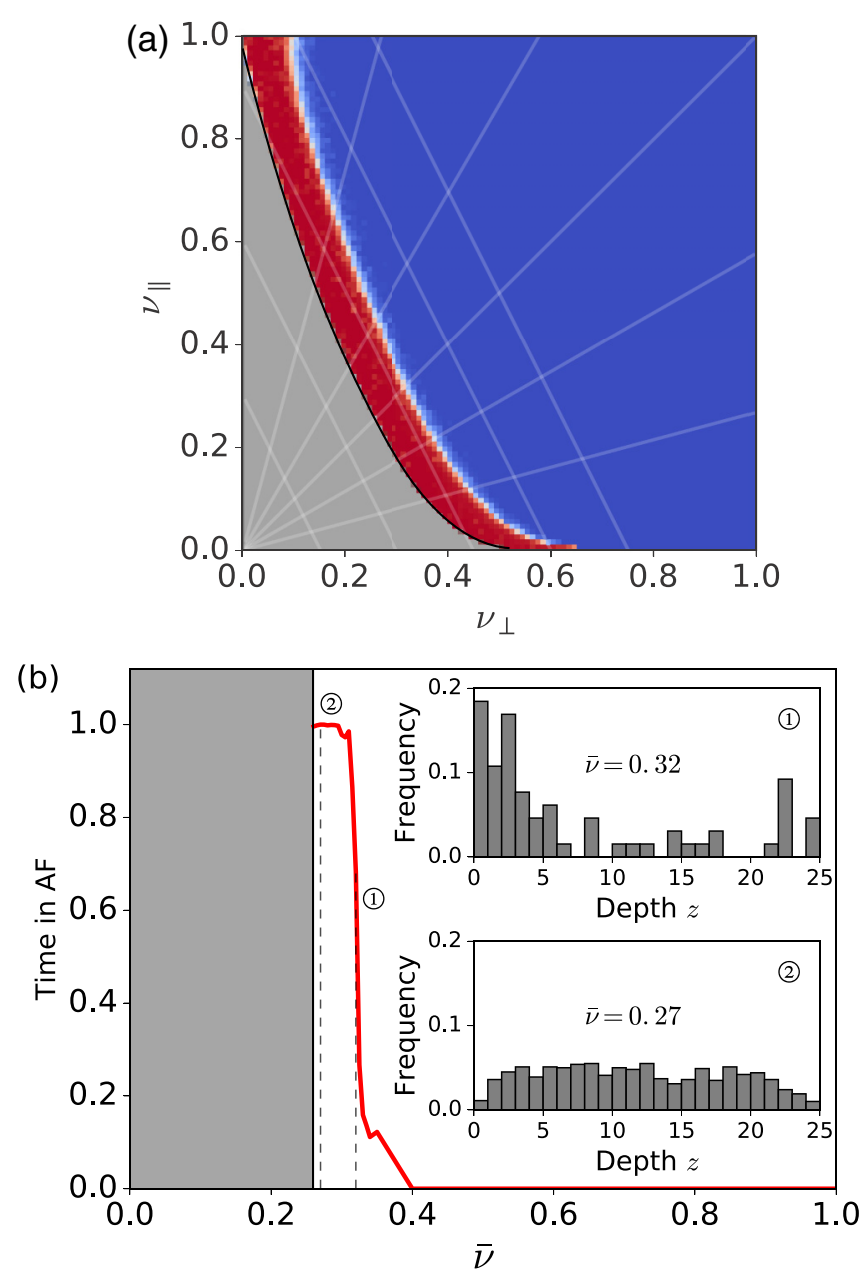

FIG. 3. The average time spent in AF for the (a) homogeneous and (b) inhomogeneous models. Both models show a transition from sinus rhythm at large coupling (0\% AF time) through paroxysmal $\mathrm{AF}(0 \%<\mathrm{AF}$ time $<100 \%)$ to persistent $\mathrm{AF}(100 \% \mathrm{AF}$ time). The gray parameter regions indicate coupling parameters below the percolation threshold, and are thus irrelevant to clinical AF. (a) Homogeneous coupling parameters, $v_{\|}$and $v_{\perp}$. Blue, sinus rhythm; red, persistent AF. The white transition region corresponds to paroxysmal AF. Guidelines of positive (negative) gradient indicate constant $\Delta \theta(\bar{v})$. Risk curve as a function of the inhomogenous coupling parameter, $\bar{v}$, (red graph). There is no AF for $\bar{v} \gtrsim 0.4$. Decreasing $\bar{v}$ associated with decoupling nodes, there is a transition from sinus rhythm through paroxysmal AF to persistent AF. Inset: Histograms showing the distribution of reentrant circuits driving AF for paroxysmal AF (top) and persistent AF (bottom) as a function of depth $z$ from the endocardium. Drivers cluster in the subendocardial region $(z=0)$ for paroxysmal $\mathrm{AF}$ but are uniformly distributed across the bulk for persistent AF.

the tissue is independent of the tissue elsewhere in the model. Therefore, the results are robust against different choices of fiber orientation and tissue thickness that may be associated with the left or right atria [40], and show that reentrant circuits will first emerge on the surface with the strongest longitudinal coupling (see Figs. 17 and 19 in Appendix D).

Visualizing the activation patterns in the inhomogeneous model on the surfaces at $z=0$ (endocardium) and $z=24$
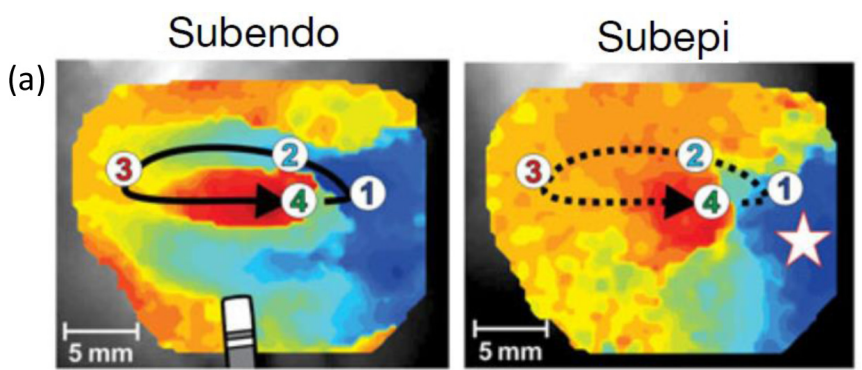

(b)
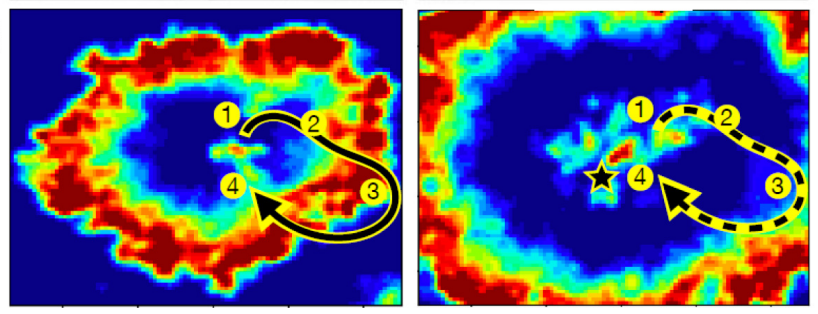

FIG. 4. (a) An activation map adapted with permission from Ref. [14] showing reentry activity from subendo imaging and a focal source on the subepi side with four marked reference points. (b) The equivalent for our model. Smoothing has been applied to images for clarity. Red, excited nodes; blue, resting nodes; other, refractory nodes.

(epicardium), we observe activation patterns consistent with Ref. [14]. In Fig. 4, the left (right) panel shows the activity when viewed from the endocardium (epicardium). For the activation maps, electrical activity spreads across the tissue from point 1 through point 2 , but is blocked from reaching the isolated fiber at point 4 . The activity loops around the isolated fiber and reenters the fiber at point 3. This back-propagating excitation passes through point 4 before reexciting point 1 , which sustains the reentrant circuit. Viewing the same region from the epicardium does not show the same reentry activity. Instead, the excitation emerges as a point source indicated by a star. The surface activity driven from the atrial bulk will typically appear as a breakthrough point, as opposed to reentry activity. A cross section of the activation patterns from endoto epicardium is shown in Fig. 2.

Figure 5 shows the global and local success rate of ablation in the model. Globally, Fig. 5(a) indicates that the success of ablation exhibits phase transition-like behavior. At low coupling, focal ablation consistently fails to terminate fibrillation, independent of the number of ablation lesions. Above a transition coupling value, the ablation success rate rapidly increases with increased coupling. The success rate is higher if multiple ablations are applied. The results indicate that focal ablation is increasingly ineffective as AF becomes more persistent. However, despite the fact that ablation fails globally, Fig. 5(b) indicates that, locally, ablation does successfully destroy the targeted reentrant circuits if the ablation is transmural. For nontransmural ablation, ablation has a much higher local success rate at high coupling values - half depth ablation has a success rate of $90 \%$ (50\%) at high (low) coupling. A direct comparison between transmural and nontransmural ablation is shown in Fig. 6. The activation maps shown demonstrate that for reentrant circuits anchored to the endocardium a shallow ablation is sufficient to destroy the reentrant circuit. However, for reentrant circuits anchored to the epicardium, 

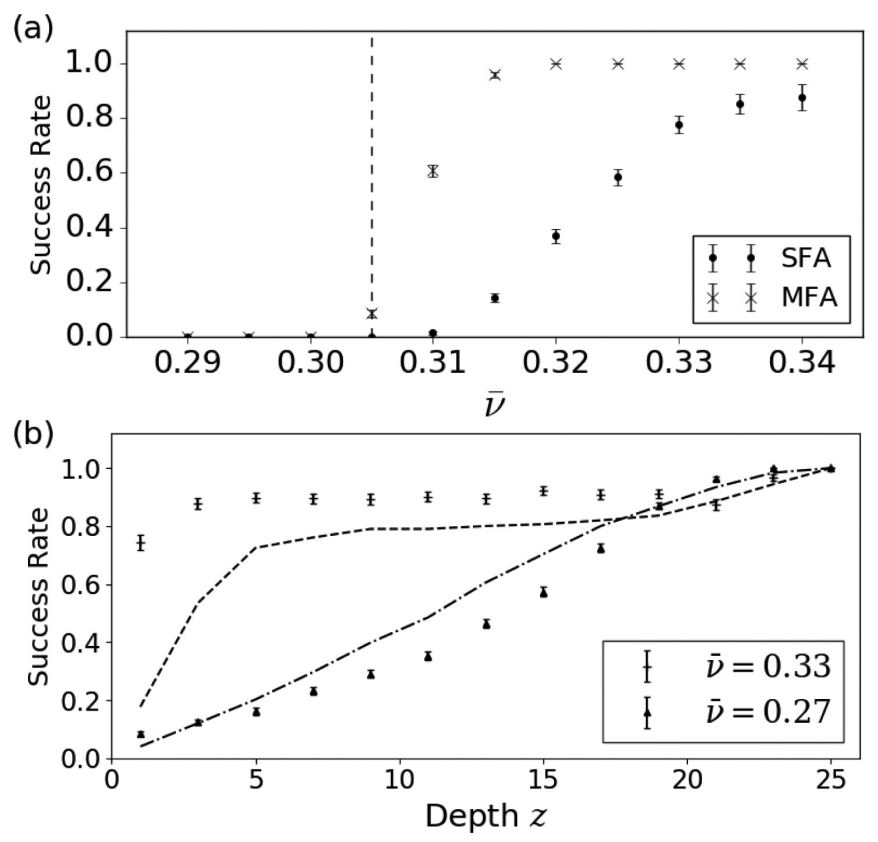

FIG. 5. (a) The global success rate of full depth focal ablation if the first reentrant circuit location is destroyed [single focal ablation (SFA), circles], and if the first ten locations are destroyed [multiple focal ablations (MFA), crosses] as a function of the coupling parameter, $\bar{v}$. The dashed line indicates the coupling value below which all simulated tissues harbour reentrant circuits. (b) The local success rate of a single focal ablation destroying reentrant circuits at the ablation site as a function of the ablation depth, $z$, for tissues with high coupling, $\bar{v}=0.33$ (plus), and low coupling, $\bar{v}=0.27$ (up triangle). The dashed (dot-dashed) lines correspond to the cumulative sum of the reentrant circuit depth probabilities for $\bar{v}=0.33$ $(\bar{v}=0.27)$.

nontransmural ablation may fail to destroy the reentrant circuit driving AF.

In Fig. 3(a), there are percolation-type transitions [42] associated with the black and white curves, respectively. The black curve outlines the bond percolation threshold for the fiber network itself: Below (above) the black curve, the fiber network does not (does) percolate from $x=0$ to $L_{x}$. This transition is irrelevant for clinical AF but the other transition is highly relevant. The white curve outlines the transition where the finite surfaces associated with missing bonds (known as hulls in three dimensions or holes in two dimensions [43]) have a linear dimension larger than or equal to $\tau / 2$ - these hulls are needed to form an isolated fiber. A fingerprint of the latter transition is also seen in Fig. 5(a). Below (above) the vertical dashed line at $\bar{v} \approx 0.305$, the global success rate of multiple focal ablations is zero (nonzero). In an infinite lattice, this corresponds to the number of finite hulls with linear dimension $>\tau / 2$ being infinite (finite) slightly below (above) this threshold.

\section{DISCUSSION}

These model observations are directly compatible with recent clinical findings that identified rotational activity from endocardial mapping in patients with paroxysmal AF [12,39], and another clinical observation identifying focal activity in patients with persistent AF from epicardial mapping [11]. Moreover, our model offers a natural explanation why ablation is more successful for paroxysmal than persistent AF. Studies have shown that ablation lesions become smaller as they penetrate further into the tissue from the endocardial surface, and that ablation struggles to penetrate more than $2 \mathrm{~mm}$ into the atrial wall [41], which can be up to $7 \mathrm{~mm}$ thick [14]. Hence, because the average driver position moves deeper into the atrial wall as AF becomes more persistent, ablation lesions need to be more accurately positioned and they must penetrate further into the tissue, restricting ablation efficacy. The model suggests that if the atria are sufficiently fibrotic, reentrant circuits are so numerous that focal ablation cannot terminate AF, even with multiple ablation lesions [see Fig. 5(a)].

The model may explain the prevalence of paroxysmal $\mathrm{AF}$ in the pulmonary veins (PVs), where sleeves of cardiac tissue extend into the PVs [20,44]. The sleeves get thinner further into the PVs and there are significant changes in the fiber orientation from the endo- to epicardium [45-47]. These physiological changes reduce cell-to-cell coupling, which the model has shown is the key requirement for forming the reentrant circuits that drive AF. Intriguingly, another recent study also demonstrates transmural reentry, specifically in the PVs, under the assumption of isotropic conduction velocities [48].

\section{LIMITATIONS AND NEXT STEPS}

Our model is much simpler than alternative biophysically realistic models [24,34]. We do not account for rate dependent effects in the action potential propagation, although we expect this to play a minimal role in the development and maintenance of structural reentrant circuits. This has the benefit that we are able to perform unparalleled statistical analyses to fully explore the differences between paroxysmal and persistent $\mathrm{AF}$, but at the cost of electrophysiological realism. As a result, our model focuses purely on the initiation and maintenance of AF from microanatomical reentrant circuits and not on AF initiated or maintained by other methods such as cardiomyocyte automaticity or the formation of rotors and scroll waves. These mechanisms are best studied using alternative models.

In our model, we consider general structural properties such as the difference in fiber orientation between the endoand epicardium, but we do not explicitly account for specific anatomical fiber bundles in the real heart or differences between the left and right atria. Therefore, with the current framework, we cannot explain why local drivers are found more frequently in the left atrium than in the right atrium $[10,13]$. However, in a proof of concept work where we have adapted the current methods for use with a real atrial fiber map, reentrant circuits do appear to form preferentially in the left atrium [49]. Additionally, we observe circuits forming in a number of key locations suspected for their role in the formation of local drivers in persistent $\mathrm{AF}$, including the sleeves of the pulmonary veins, the posterior atrial wall, and the atrial appendages [50].

To address these limitations and verify the results presented here, we propose a potential experimental test and a number of suggested model developments. First, adapting the model to a realistic atrial topology with an integrated fiber structure will 


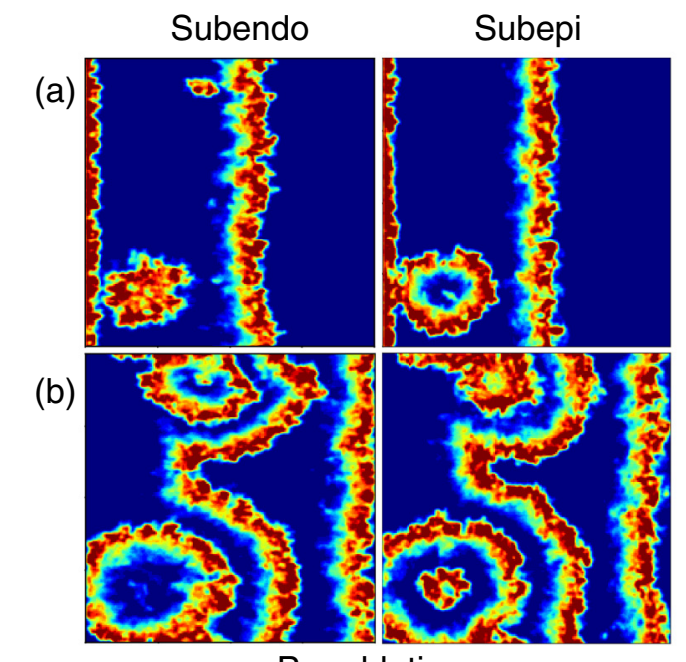

Pre-ablation
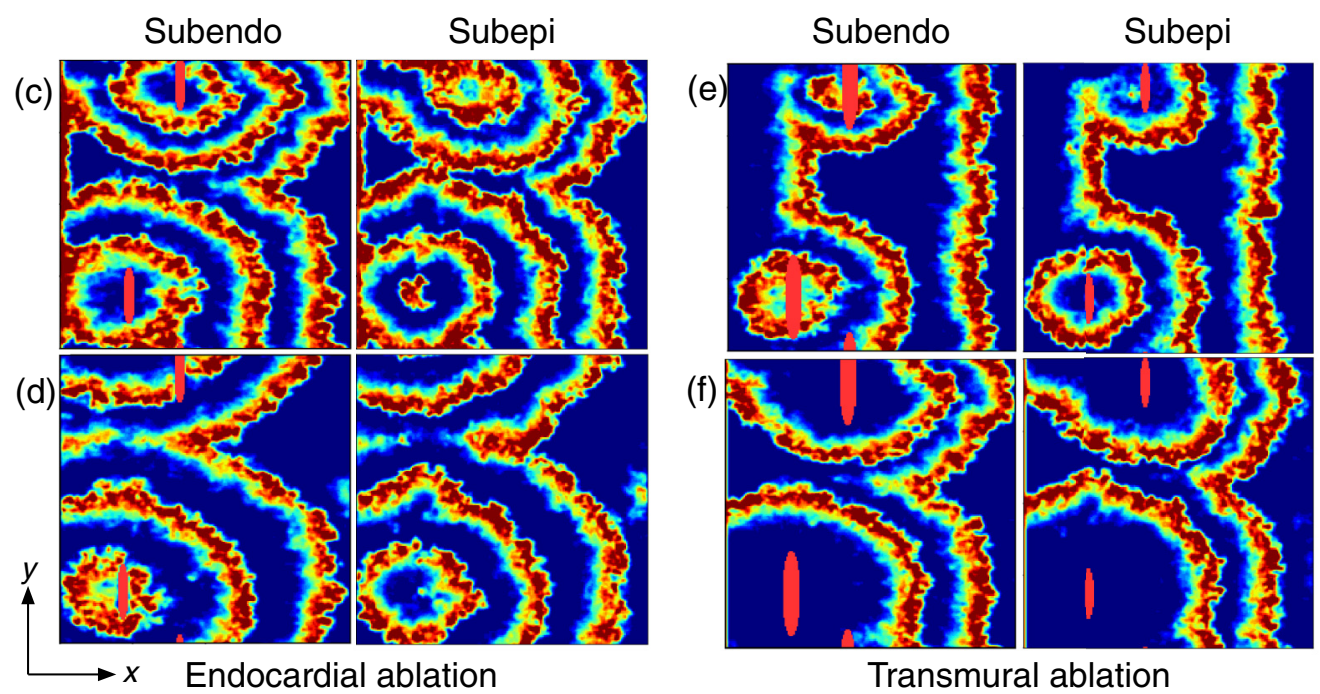

Transmural ablation

FIG. 6. Simulated ablation in a tissue with multiple drivers demonstrating how the ability to terminate fibrillatory activity is closely related to driver depth. AF emerges (a) in the bottom left corner from a reentrant circuit near the epicardium, and (b) at the top of the tissue from a separate reentrant circuit near the endocardium. (c) Two shallow ablations (solid red in bottom left and top center) are applied to the endocardial surface at the locations of the reentrant circuits penetrating part of the way into the tissue. (d) The circuit near the epicardium has not been destroyed by the ablation since the ablation has not penetrated far enough into the tissue. The circuit near the endocardium has been destroyed successfully. However, since an active circuit remains in the tissue, AF has not been terminated. (e) and (f) show the same ablation process as demonstrated in (c) and (d); however, the ablation is now transmural, penetrating the full distance through the tissue. This is indicated by the solid red regions on the subepi view in (e) and (f). The ablations have successfully destroyed both reentrant circuits. Hence, fibrillatory waves dissipate as shown in (f) and sinus rhythm will be restored. Note, transmural ablation is not always possible in clinical practice. Ablation lesions typically struggle to penetrate more than $2 \mathrm{~mm}$ [41] into the atrial wall, which can be up to $7 \mathrm{~mm}$ thick in places [14]. Scale: $200 \times 200$ nodes (full tissue).

allow us to refine the predictions made here and make concrete statements about different regions of the atria and specific anatomical structures. This includes investigating which atrial regions are especially prone to the formation of reentrant circuits and testing whether these correlate with known clinical data. We have taken the first steps to adapt the current model to a realistic atrial fiber structure in [49]. Currently, both the model presented here and the adapted model consider the role of local decoupling of muscle fibers, simulating the action of diffuse interstitial fibrosis or fatty infiltration. However, more pronounced global decoupling may occur in regions with compact fibrotic lesions. Future versions of the model should consider a full range of potential fibrosis patterns when analyzing the emergence of reentrant circuits.

As a potential experimental verification of our results, we propose following the methods set out in Ref. [14]. Using explanted Langendorff-perfused animal hearts with a diffuse fibrosis model at different levels of fibrotic burden, simultaneous endo- and epicardial optical mapping may be used to assess how the distribution and depth of microanatomical reentrant circuits change with increasing fibrotic burden. As the fibrotic burden is increased, our model would predict an 
increase in the number of observed drivers, and a reduction (increase) in the ratio of reentrant drivers to focal drivers seen on the endocardium (epicardium).

\section{CONCLUSIONS}

The overarching aim of this paper was to create the simplest possible bioinspired model that displays the variety of clinically observed activation patterns [14] and can exploit large scale statistics to make concrete predictions not accessible in a laboratory setting.

The model is consistent with the typical evolution of $\mathrm{AF}$ from sinus rhythm through paroxysmal AF to persistent AF. Moreover, combined with the clinical observations in Ref. [14], our model gives substantial evidence for the proposed unifying mechanism of local drivers in AF, suggesting a potential resolution to long-standing debates. Complementing this finding, we predict that the inhomogeneity in fiber orientation causes the average depth of drivers to move away from the endocardium and into the bulk of the atrial wall as AF becomes more persistent, potentially explaining why ablation is less successful for persistent $\mathrm{AF}$ than for paroxysmal $\mathrm{AF}$, if experimentally verified. This insight is the first of its kind and may have key clinical implications.

Work is currently underway on adapting the model to realistic atrial fiber maps with the hope that this may be able to identify reentrant circuit risk regions clinically on a personalized basis [51,52].

\section{ACKNOWLEDGMENTS}

M.F. thanks Timotej Kapus and Éamonn Murray for computational support, and Tim S. Evans for helpful comments. M.F. and A.C. gratefully acknowledge PhD studentships from the Engineering and Physical Sciences Research Council through Grants No. EP/N509486/1 and No. EP/L015129/1 respectively. N.S.P. acknowledges funding from the British Heart Foundation (Grant No. RG/16/3/32175 and Centre for Research Excellence RE/18/4/34215) and the National Institute for Health Research Biomedical Research Centre. K.C. and N.S.P. acknowledge funding from the Rosetrees Trust Grant A1173/M577.

K.C. conceived the original model. M.F. proposed the connection to Ref. [14] and the investigation into the success of ablation, and adapted the model for variable fiber orientation. A.J.F. and A.C.L. implemented the model and ran simulations, supervised by M.F.. R.L. simulated the effect of ablation in the model. A.C. developed the theoretical aspects of the model. N.S.P. provided the medical expertise for the project. K.C. supervised all aspects of project. All authors contributed to the writing of the paper.

\section{APPENDIX A: MODEL DEFINITION}

\section{Heart muscle structure}

The area of the atrial muscle is $L_{x}^{\prime} \times L_{y}^{\prime} \approx 20 \mathrm{~cm}^{2}[53,54]$ while the thickness of the atrial muscle wall ranges from 1 to $7 \mathrm{~mm}$ [14]. A typical average is about $L_{z}^{\prime}=2.5 \mathrm{~mm}$ [55]. The atrial muscle tissue comprises tubular shaped heart muscle cells (myocytes) of length $\Delta x^{\prime} \approx 100 \mu \mathrm{m}$ and diameter $\Delta y^{\prime}=$ $\Delta z^{\prime} \approx 20 \mu \mathrm{m}[56,57]$. Cells are predominantly connected

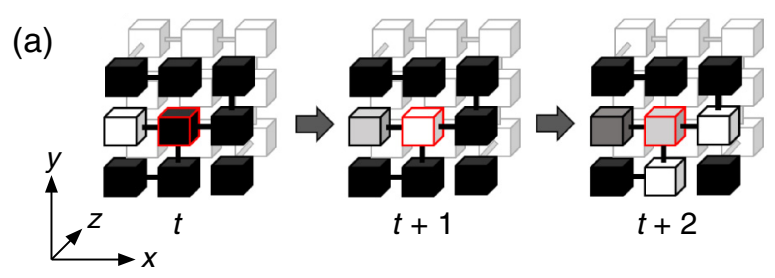

(b)

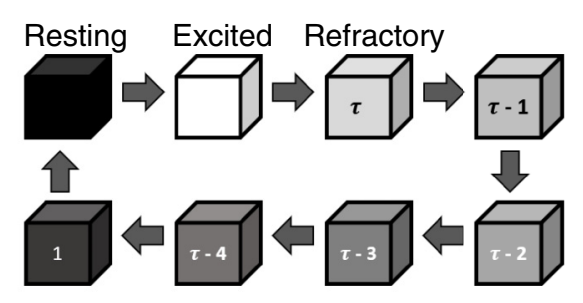

FIG. 7. (a) The excitation wave propagates across neighboring nodes. Nodes are connected longitudinally with frequency $v_{\|}$and transversely (along $y$ and $z$ directions) with frequency $v_{\perp}$. At time $t$, the red bordered node is resting. At time $t+1$, the node activates in response to the stimulus coming from its neighbor to the left. At time $t+2$, the red bordered node becomes refractory and the excitation wave activates the two nodes in the resting state to which it is coupled. The node to the left of the red bordered node cannot be excited as it is in a refractory state. (b) Temporal dynamics of a node. Each node sequentially passes across three distinct states: resting (black), excited (white), and refractory (gray scale).

longitudinally, forming fibers that connect transversely now and again. Hence a branching network of anisotropic cells provides an appropriate approximation of the atrial tissue.

\section{Heart muscle physiology}

The membrane potential of heart muscle cells shows that they can be in one of three states: excited, where the cell depolarizes; resting, where the cell can be depolarized by a neighboring active cell; and refractory, where for some time $\tau^{\prime}$ after excitation the cell cannot be reexcited. The shortest time scale is associated with a cell depolarizing, $\Delta t^{\prime} \approx 0.6 \mathrm{~ms}$, whereas the refractory period lasts $\tau^{\prime} \approx 150 \mathrm{~ms}$ under rapid pacing. When a resting cell is induced to excite by a neighboring excited cell, it contracts. The propagation speed of the activation wavefront is $\Delta x^{\prime} / \Delta t^{\prime} \approx 0.2 \mathrm{~ms}^{-1}$ and the heart's natural pacemaker (sinus node) ensures the periodic propagation of a coordinated activation wavefront, causing atrial muscle contractions. The rate of excitations is controlled by the pacemaker cells that regularly self-excite with period $T^{\prime}=300-1000 \mathrm{~ms}$. For example, $T^{\prime}=660 \mathrm{~ms}$ corresponds to approximately 90 beats per minute.

\section{Dimensionless parameters of the model}

We can translate real tissue values into dimensionless model parameters. We apply a similar conversion procedure to that presented in Ref. [23]. The dimensions of the simplified 3D topology are $L_{x}=L_{x}^{\prime} / \Delta x^{\prime}=1000, L_{y}=L_{y}^{\prime} / \Delta x^{\prime}=1000$, and $L_{z}=L_{z}^{\prime} / \Delta z^{\prime}=125$. We apply a coarse grain transformation $\Delta x^{\prime} \rightarrow b \Delta x^{\prime}, \Delta y^{\prime} \rightarrow b \Delta y^{\prime}, \Delta z^{\prime} \rightarrow b \Delta z^{\prime}$ such that we 
(a)

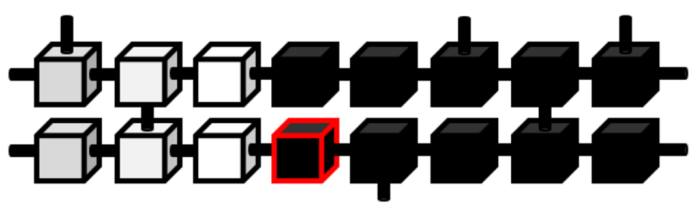

(b)

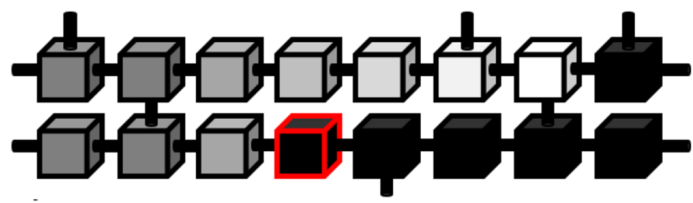

(c)

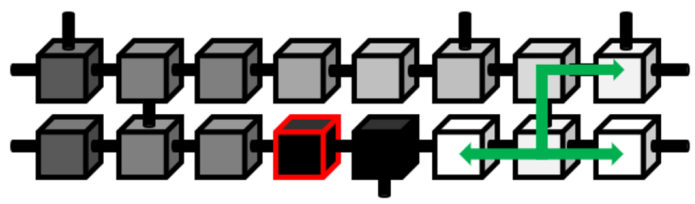

(d)

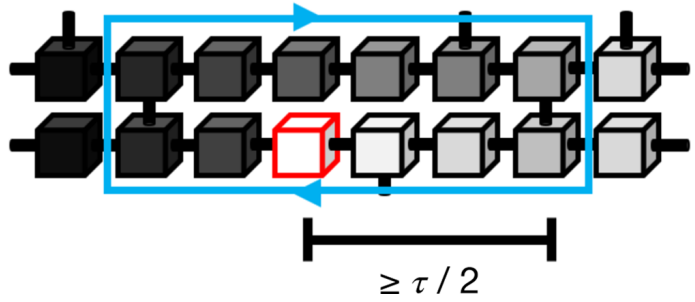

FIG. 8. Progression of the excitation wave through a simple reentrant circuit situated on the surface of the 3D topology used to mimic the heart muscle fiber network. The red bordered node is susceptible to conduction block. ( $a, b)$ The planar excitation wave reaches the node that is susceptible to conduction block. The red bordered node does not excite and blocks the propagation in the lower fiber while the planar propagation of the wavefront proceeds in the upper fiber. $(b, c)$ The activation wavefront reaches the penultimate node in the upper fiber that connects the two adjacent fibers. The excitation advances in multiple directions, indicated by the green arrows. In particular, the wavefront reenters the fiber where the original conduction was blocked. (c, d) If the path length indicated in the lower fiber is longer than $\tau / 2$, the node placed to the left of the red bordered node will have reached its resting state when the backward propagation arrives. Therefore, the region indicated in light blue will act as a reentrant circuit until the red bordered node blocks the excitation again. The likelihood of creating a reentrant circuit is determined by the coupling frequencies $v_{\|}$and $v_{\perp}$ and the fraction $\delta$ of nodes susceptible to conduction block.

group $b^{3}$ cells within one node of the model. The propagation speed has to remain constant $\Delta x^{\prime} / \Delta t^{\prime} \approx 0.2 \mathrm{~ms}^{-1}$ so we must also coarse grain time $\Delta t^{\prime} \rightarrow b \Delta t^{\prime}$ [23]. By using $b=5$, we find the dimensionless parameters of the model. For the 3D pipe structure, we have $L_{x}=L_{x}^{\prime} /\left(b \Delta x^{\prime}\right)=200, L_{y}=$ $L_{y}^{\prime} /\left(b \Delta y^{\prime}\right)=200$, and $L_{z}=L_{z}^{\prime} /\left(b \Delta z^{\prime}\right)=25$. The renormalized excitation time $b \Delta t^{\prime}=3 \mathrm{~ms}$ yields a dimensionless refractory period $\tau=\tau^{\prime} /\left(b \Delta t^{\prime}\right)=50$ and pacemaker activation period $T=T^{\prime} /\left(b \Delta t^{\prime}\right)=220$, assuming $T^{\prime}=660 \mathrm{~ms}$.

\section{Modeling the real atrial tissue}

The structural properties are mimicked by a 3D pipelike topology of the atria, $L_{x} \times L_{y} \times L_{z}$, where $x$ is the longitudinal direction and $y$ and $z$ are the transverse directions. Given that the mechanism of reentrant circuit formation in the model is fully local, the precise topology chosen for the model has a

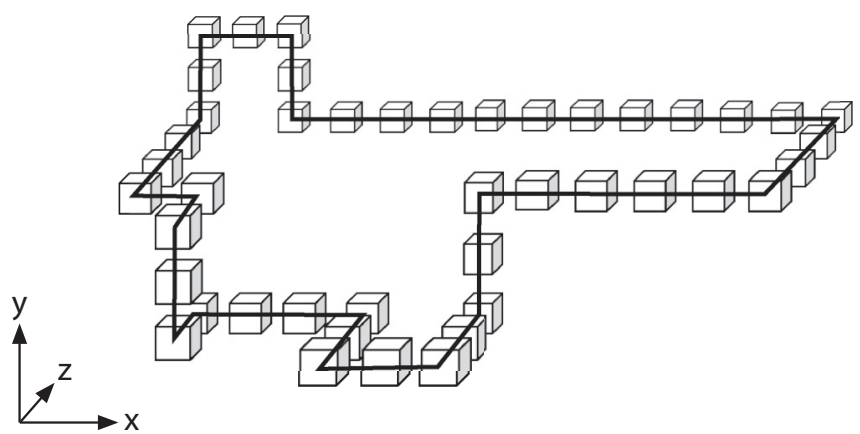

FIG. 9. Three-dimensional visualization of an AF driver located in the bulk of the atrial tissue. The black path represents the connectivity structure between the nodes. In this particular example we observe that longitudinal connections are more frequent than transverse connections ( $y$ and $z$ directions), representative of reentrant circuits that can be observed when $v_{\|}$is substantially larger than $v_{\perp}$.

minimal effect on the results. The pacemaker cells are the nodes at the boundary $x=0$ and we apply open boundaries in $x$ and $z$ and periodic boundaries in $y$. Each of the $L_{z}=25$ layers contain $L_{x} \times L_{y}=200 \times 200$ nodes. The nodes are connected longitudinally with frequency $v_{\|}$and transversely (within the layer and across the layers) with frequency $v_{\perp}$. The network of nodes mimics the branching structure of heart muscle fibers and it is kept fixed for a given realization.

To mimic the membrane potential of a cell, nodes can be in one of three distinct states: resting, excited, or refractory. At a given time $t$, an excited node induces resting nodes to which it is coupled to excite at time $t+1$. The excited node at time $t$ becomes refractory at time $t+1$ and it will not be able to respond to any further stimulus during the next $\tau$ time steps.
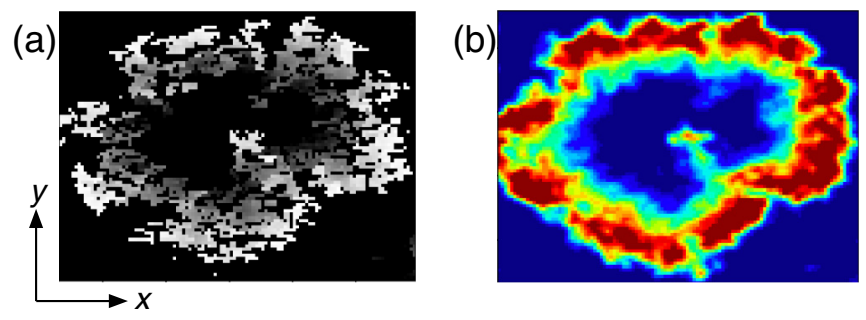

FIG. 10. Surface activation patterns in the model (a) before and (b) after processing. (a) The raw node states of a single layer of nodes in the model. White indicates active nodes, black nodes are resting (excitable), and gray nodes are refractory. This raw image only shows the node states in a single layer at a fixed $z$ coordinate. (b) To aid data interpretation and match the visualization methods applied in Ref. [14] we process the raw image using Gaussian smoothing. A 3D Gaussian kernel is used to convolve the raw node states. The Gaussian is centered on the imaged surface with standard deviations $\sigma_{x}=\sigma_{y}=1$ and $\sigma_{z}=5$. The color scheme for the activation maps after processing has been chosen to match that used in Ref. [14]. Active wavefronts are shown in red, resting nodes are in blue, and refractory nodes are represented by all other colors. This filtered image improves the coherence of wavefronts in the model and aids data interpretation. Scale: $100 \times 75$ nodes. 
(a)

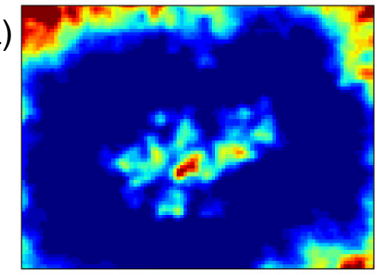

(c)
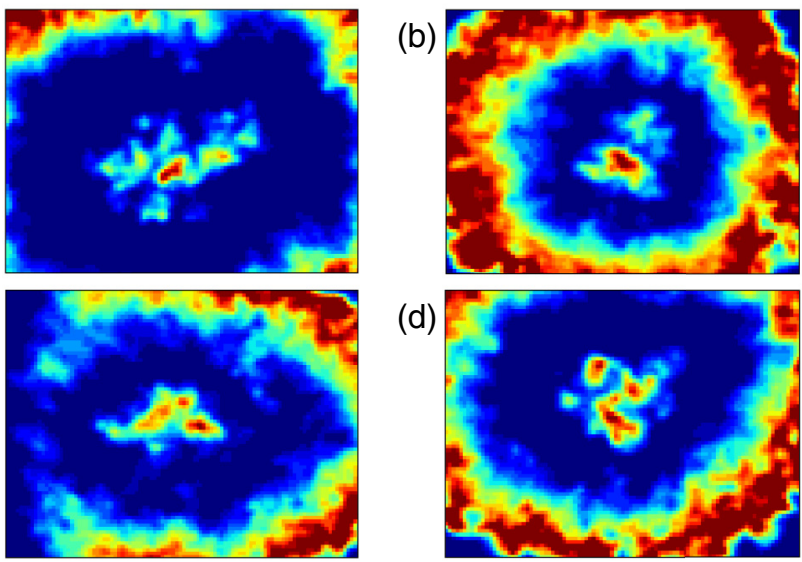

(d)

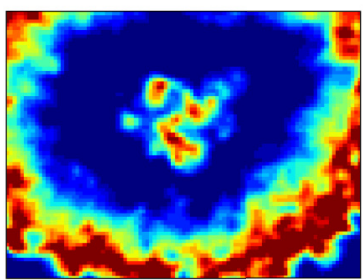

(e)

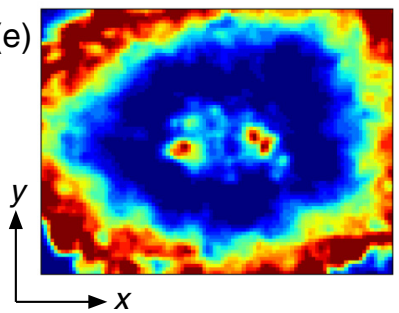

FIG. 11. Examples of breakthrough patterns in the model. Closeup view of activity for five different realizations (tissues). Tissues (a) and (b) show one primary breakthrough point. Tissues (c) and (d) show the activity emerging across a wider area almost simultaneously. Tissue (e) shows two clearly divided breakthrough points. Globally, all five tissues have breakthrough activity that will generate waves that appear to originate from a focal point. These activation patterns are typically observed on the epicardium during paroxysmal $\mathrm{AF}$, but may, in rare cases, also be seen on the endocardium. Quantitatively differentiating between breakthrough types is difficult. See videos $\mathrm{A}, \mathrm{B}, \mathrm{C}$, and E. Scale: $100 \times 75$ nodes.

The refractory node will revert to a resting node after $\tau$ time steps (see Fig. 7).

A small fraction $\delta$ of the nodes can be susceptible to conduction block. These nodes are selected at random and their position in the network is fixed for a given realization. These particular nodes will, with a small probability $\epsilon$, fail to activate when their neighbors excite. In a well connected
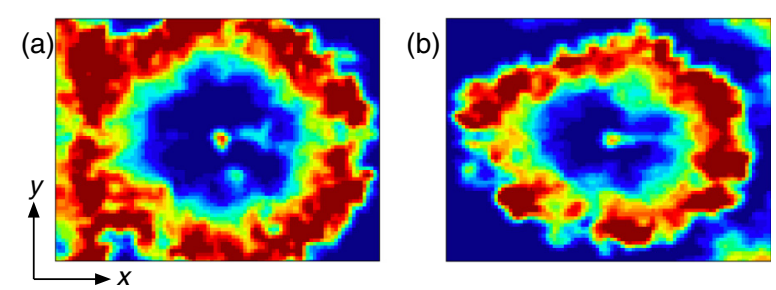

FIG. 12. Examples of partial and full reentry in the model. (a) An example of partial surface reentry where a full rotation is not observed in the surface activity. This is because the reentrant circuit stretches to a depth of approximately four nodes from the endocardial surface. (b) Complete surface reentry, where no gaps are observed in the reentrant circuit. Reentrant circuits are typically observed on the endocardium during paroxysmal AF, but may also be found on the epicardium in rare cases. See video D. Scale: $90 \times 70$ nodes.
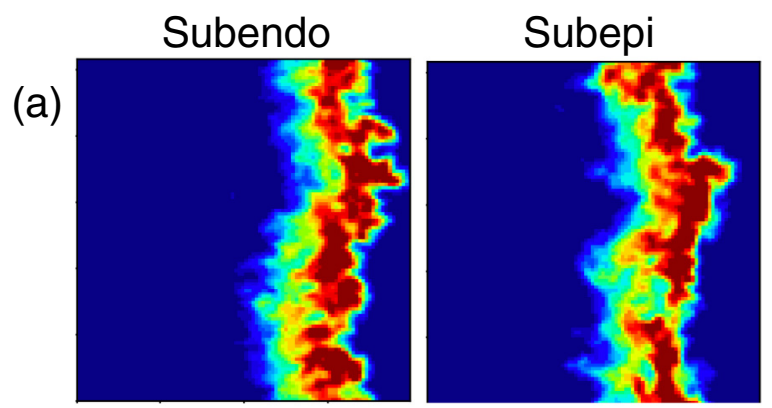

(b)
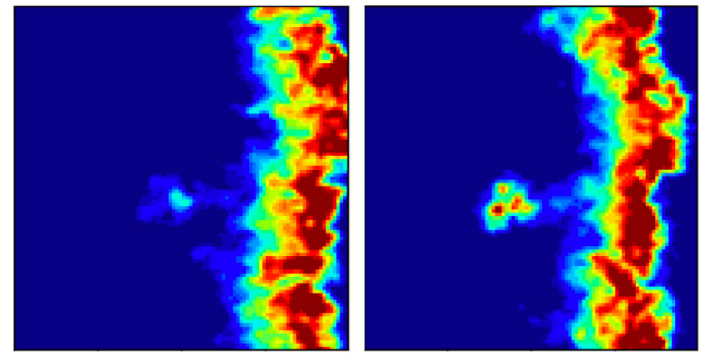

(c)
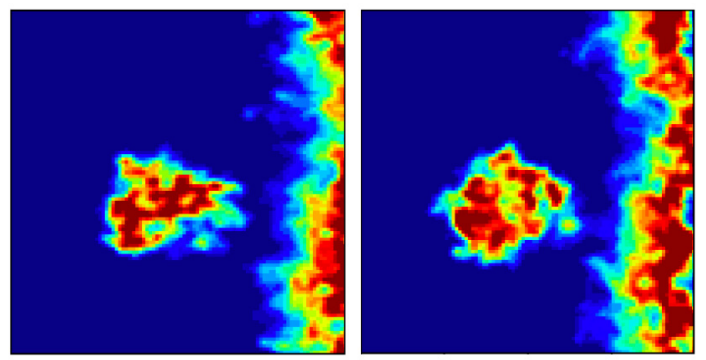

(d)
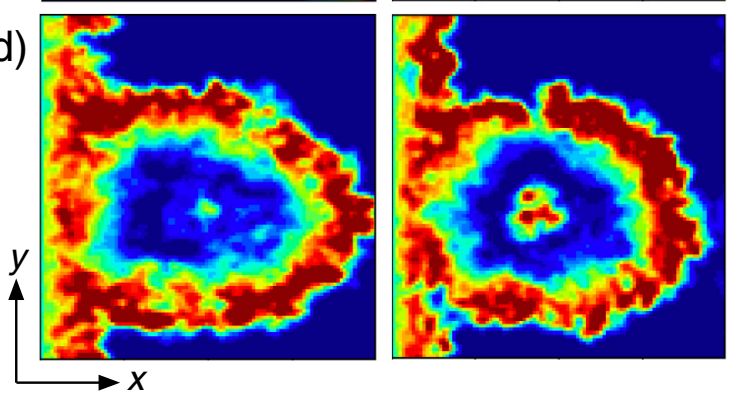

FIG. 13. An example of surface activation patterns where reentrant activity is not observed on either atrial surface. Close-up view of the activation pattern in the case of a reentrant circuit with depth $z=16, \bar{v}=0.31$. (a) The tissue in sinus rhythm with regularly propagating wavefronts. A signature of fibrillatory activity characterizing AF is not observed. (b) Initial fibrillatory activity emerges on both epicardium and endocardium as breakthrough points. Note that this reentrant circuit is eight layers closer to the epicardium than the endocardium, as can be seen by the delay between activation patterns. (c) Fibrillatory activity spreads across the tissue. (d) AF is sustained. Reentrant circuits are not observed on either surface. Scale: $100 \times 100$ nodes.

network, such a failure will not disrupt the planar propagation of the excitation wave and sinus rhythm prevails. However, if the network of heart muscle cells becomes too decoupled, for example due to fibrosis, then, when a failure occurs, the planar propagation of the excitation wave may be disrupted. The break of the regular wavefront may prompt the spontaneous emergence of circuits formed by wavelets of excitation leaking back through the refractory wake of the 


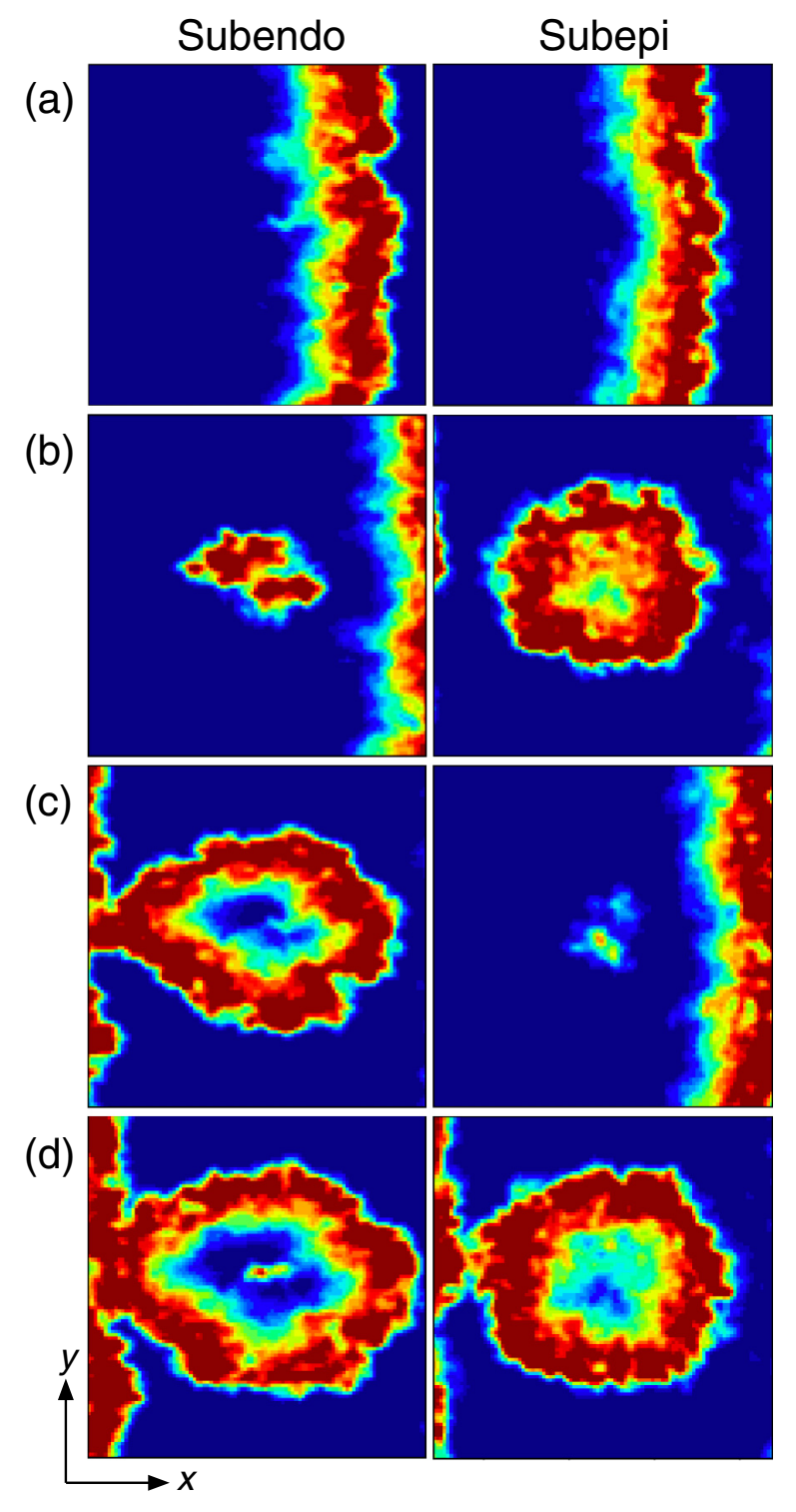

FIG. 14. An example of reentrant activity observed on the endocardial surface. Close-up view of activation patterns in the case of a reentrant circuit near the endocardial surface with depth $z=1$, $\bar{v}=0.33$. (a) The model in sinus rhythm. A faint reentrant excitation is observed at the center of the subendo view. (b) Fibrillatory activity emerges in the model. An unexcited tract on the right side of the active region in the subendo view indicates the isolated fiber for the reentrant circuit. Activity spreads on the subepi view resembling a focal source. (c) The wavefront reenters the unexcited tract in the subendo view. The activity breaks through onto the epicardial surface at a single stable breakthrough point. (d) Fibrillatory activity is sustained. Scale: $100 \times 100$ nodes.

wavefront [23]. This occurs if nodes in a certain region of the atrial muscle form a reentrant circuit (see Fig. 8). Reentrant circuits can emerge spontaneously in the atria due to structural changes during the lifetime of a patient as specific pathologies (e.g., fibrosis) might decrease the coupling between cells.

The critical structures discussed in Fig. 8 have been introduced in Ref. [23]. In the three-dimensional network, similar
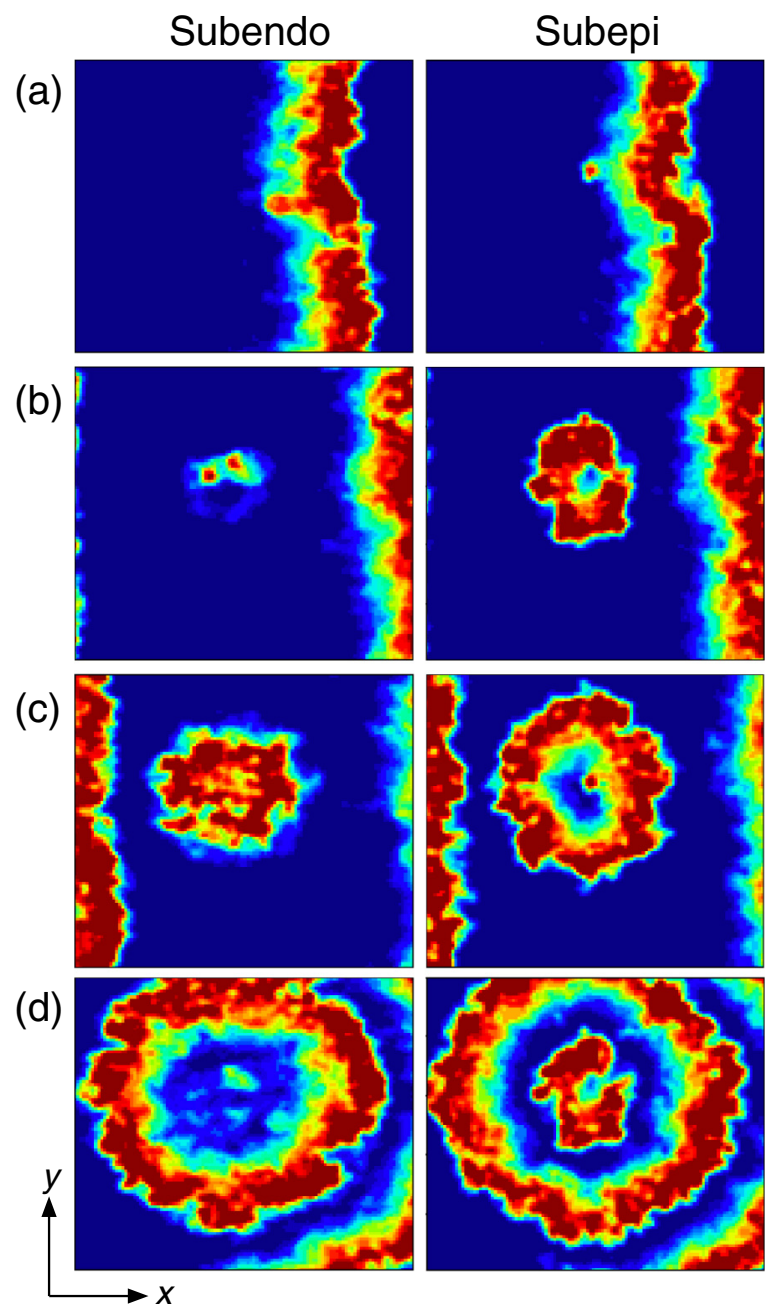

FIG. 15. An example of reentrant activity observed on the epicardial surface. Close-up view of the activation pattern in the case of a reentry circuit near the epicardium with depth $z=22, \bar{v}=0.32$. Circuits forming near the epicardium are much rarer than those forming near the endocardium in paroxysmal AF. (a) The model in sinus rhythm. A faint reentrant excitation is observed above the center of the subepi view. (b) Fibrillatory activity emerges in the model. An excitation is just starting to enter the unexcited tract on the right side of the active region in the subepi. Fibrillatory activity has broken through to the endocardial surface at multiple points simultaneously. (c, d) Fibrillatory activity is driven and sustained. A reentrant circuit is clearly observed from subepi imaging. Activity appears to originate from a focal point in subendo imaging. Scale: $110 \times 100$ nodes.

configurations are relevant but now they can be placed within the bulk as well as on the endo- and epicardial surfaces (see Fig. 9 for a simple visualization).

\section{APPENDIX B: DATA VISUALIZATION}

The raw model data contain small scale noise which obscures the overall behavior. Therefore, to effectively visualize model activity, the raw data need to be filtered in a manner mimicking real clinical imaging techniques. The optical mapping system used by Ref. [14] is recorded to a depth 

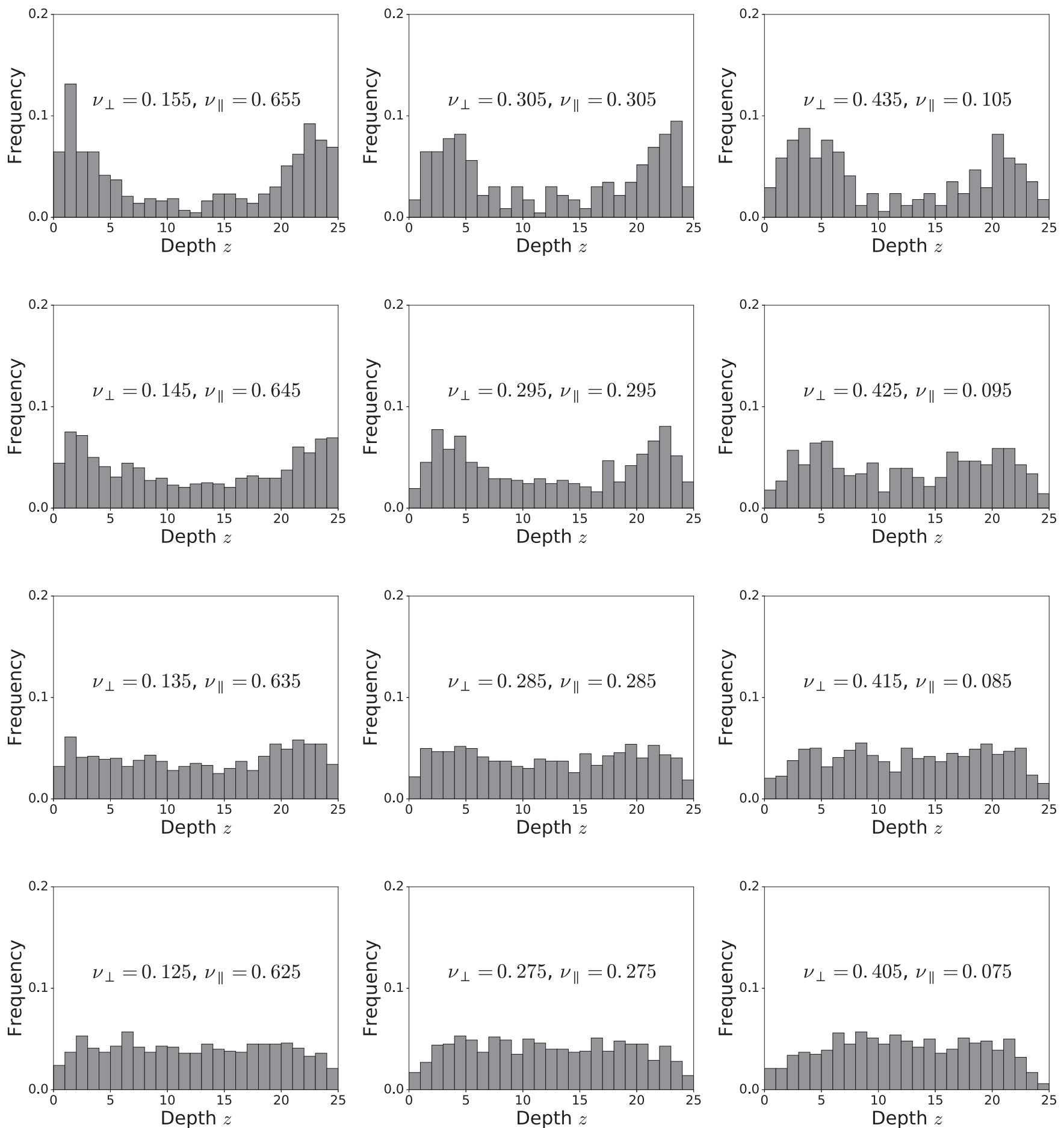

FIG. 16. The distribution of reentrant circuit depth $z$ from the endocardium in the homogeneous model. Fiber angle $\Delta \theta$ is constant across the tissue. AF progresses from paroxysmal AF (top row) to persistent AF (bottom row). The left column is for tissues with strong longitudinal coupling and weak transverse coupling. The central column is for tissues with equal longitudinal and transverse coupling. The right column is for tissues with weak longitudinal coupling and strong transverse coupling. Depth $z=0(z=24)$ corresponds to the endocardium (epicardium). In paroxysmal AF, reentrant circuits are predominantly found near the atrial surfaces. When visualized from the atrial surface, these circuits will typically appear as reentrant or rotational activity. In persistent AF, reentrant circuits are uniformly distributed in the bulk of the atrial wall, away from the epicardium and endocardium. These circuits are more likely to result in breakthrough point (focal) activation patterns when visualized from the atrial surfaces. In the homogeneous model, the endocardium and epicardium are equivalent due to the symmetry. 

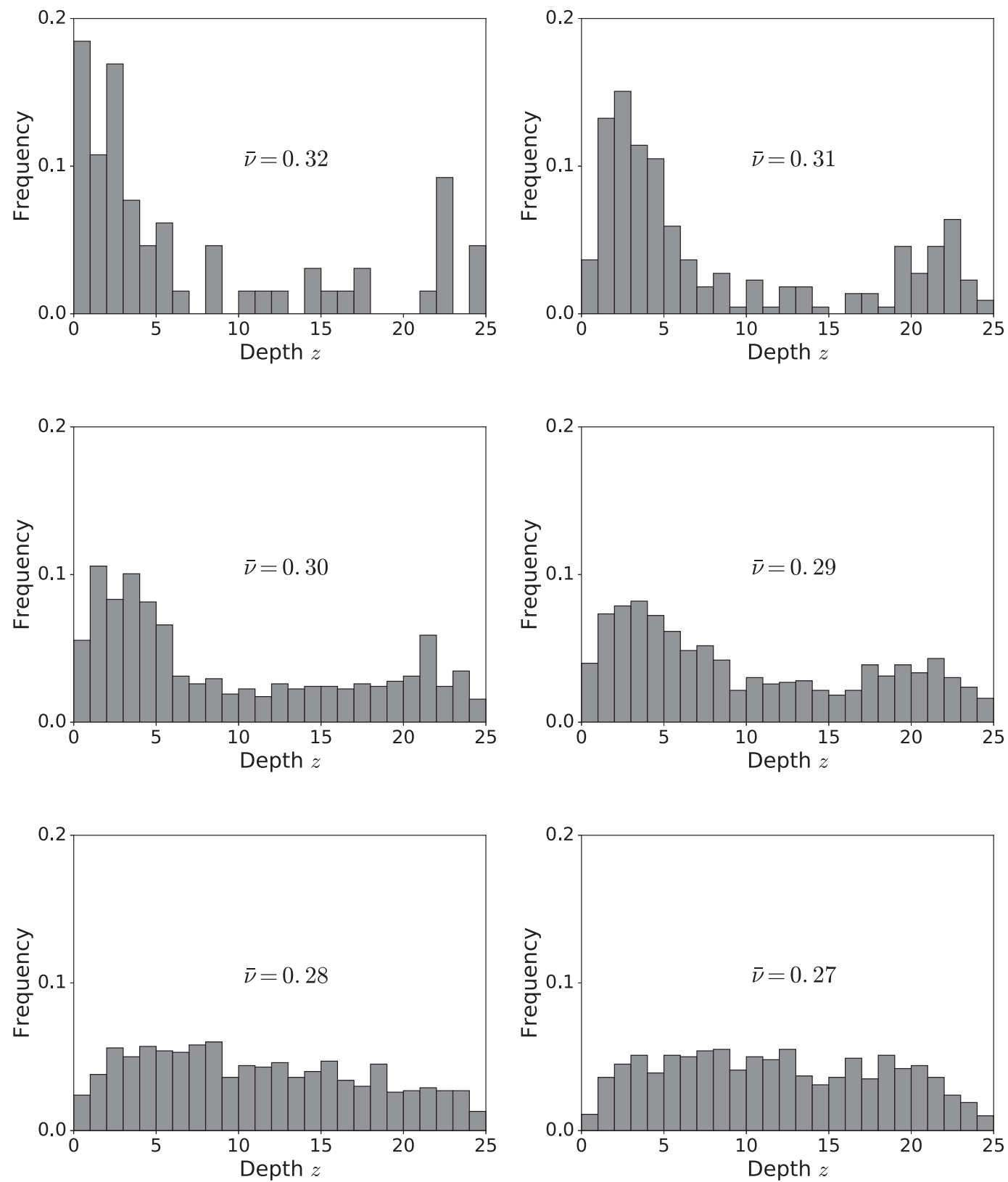

FIG. 17. The distribution of reentrant circuit depth $z$ from the endocardium in the inhomogeneous model. AF progresses from paroxysmal $\operatorname{AF}(\bar{v}=0.32)$ to persistent $\operatorname{AF}(\bar{v}=0.27)$. Fiber angle varies linearly across the tissue from $\Delta \theta_{\text {endo }}=24^{\circ}$ on the endocardium to $\Delta \theta_{\text {epi }}=42^{\circ}$ on the epicardium. Depth $z=0(z=24)$ corresponds to the endocardium (epicardium). In the inhomogeneous model, the symmetry between the epicardium and endocardium is broken with the stronger longitudinal coupling on the endocardium than the epicardium. For paroxysmal $\mathrm{AF}$, reentrant circuits are predominantly found near the atrial surfaces, and significantly more circuits cluster near the endocardium than the epicardium. This is because fibers have much stronger longitudinal coupling on the endocardial surface than on the epicardial surface. When visualized from the endocardium, these circuits will typically appear as reentrant or rotational activity. In persistent AF, reentrant circuits are uniformly distributed in the bulk of the atrial wall, away from the epicardium and endocardium. These circuits are more likely to result in breakthrough point (focal) activation patterns when visualized from the endocardium. As AF has progressed from paroxysmal to persistent, the average depth of circuits from the endocardium at $z=0$ has significantly increased-this hinders the effective use of catheter ablation for those circuits deep in the atrial bulk.

of $1-4 \mathrm{~mm}$ weighted to the layer being observed, with a resolution of approximately $0.02 \mathrm{~mm}$. These data guided the coarse graining: The activation patterns were convolved with a 3D Gaussian, with standard deviations $\sigma_{x}=\sigma_{y}=1$ and $\sigma_{z}=5$. An example of the difference between the raw and processed images is shown in Fig. 10. 
(a)

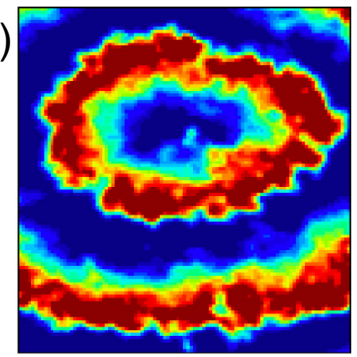

Pre-ablation

(b)
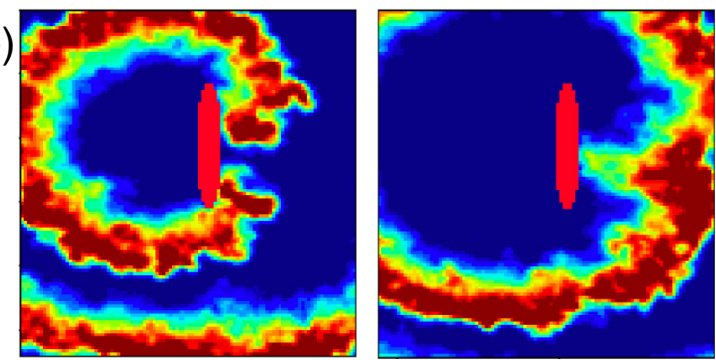

Ablation

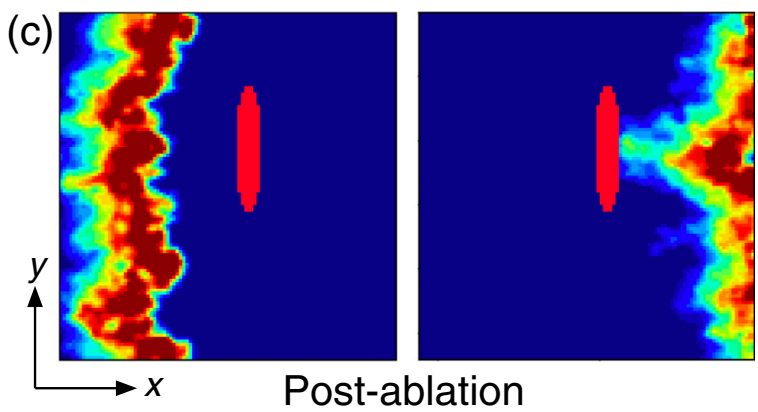

FIG. 18. The termination of AF by destroying the reentrant circuit using ablation in the inhomogeneous model. The images show the activation patterns for a close-up of the endocardial surface. (a) A faint reentrant excitation is visible just above the center of the image. This corresponds to the reentrant circuit which is driving AF. (b) Nodes surrounding the reentrant circuit are destroyed (solid red region). The reentry circuit is not sustained since the reentrant excitation collides with the ablation lesion. The fibrillatory waves dissipate. (c) The model has returned to sinus rhythm. The ablation lesion disrupts the sinus wavefront, but does not induce fibrillation. See video F. Scale: $100 \times 110$ nodes.

\section{APPENDIX C: SIMULATION DETAILS-ABLATION METHODS}

To simulate the effect of ablation in the model (results shown in Fig. 5 of the main paper) we generate 500 independent tissues for each coupling value, $\bar{\nu}$. The random seeds used to generate these tissues are saved so that the tissues can be regenerated at a later stage. An additional seed is generated for each tissue which specifies the order in which nodes susceptible to conduction block fail to excite when prompted by an excited neighbor.

For the global success rate of ablation [see Fig. 5(a) in the main paper], a simulation is started in each tissue up to a maximum time of 10000 time steps. For simulations of length 10000 ( $\approx 45$ beats), with $\epsilon=0.05$ and $T=220$, if a structure capable of generating a reentrant circuit is present, the probability it activates during the simulation is $>90 \%$. If a reentrant circuit is detected during the evolution of the simulation, the simulation is stopped and the location of the circuit is recorded. Otherwise, we mark this structure as containing no reentrant circuits and move on to the next. If a reentrant circuit is detected, using the saved seeds, the exact same tissue is regenerated and we reset the order in which nodes susceptible to conduction block fail to fire. Before the simulation is restarted, a cuboid of side length 20 nodes and depth 25 nodes, centered on the $x$ and $y$ coordinates identified as the circuit location, is destroyed, mimicking a focal ablation. For a tissue of dimensions $200 \times 200 \times 25$ nodes, this ablation lesion corresponds to $1 \%$ of the tissue. Destroyed nodes are permanently disabled and cannot be excited by neighboring excited nodes. After the ablation lesion is applied, we restart the simulation for another 10000 time steps and observe whether another reentrant circuit emerges at a different location in the tissue. If yes, this region can be ablated and the process can be repeated an arbitrary number of times-at this stage the focal ablation has been unsuccessful at terminating AF globally. If no, the ablation is considered successful and we move onto the next tissue.

The global ablation success curves shown in Fig. 5(a) of the main paper correspond to the percentage success that ablation prevents the emergence of $\mathrm{AF}$ anywhere in the tissue after a single ablation has been applied, and after up to ten individual lesions have been applied.

For the local success rate of ablation [see Fig. 5(b) in the main paper], we also generate 500 random tissues for each coupling value and save their seeds. Likewise, we save the seeds giving the sequence of failures for the nodes susceptible to conduction block. For each tissue, we run a simulation for up to 10000 time steps. If a reentrant circuit is not identified we move onto the next tissue. If a circuit is identified, a cuboid of side length 20 nodes and depth $z$ nodes, centered on the $x$ and $y$ coordinates identified as the circuit location, is destroyed. Additionally, all nodes susceptible to conduction block with coordinates outside the $x$ and $y$ extent of the ablation lesion are prevented from exhibiting unidirectional conduction block. Hence, when the simulation is restarted, a reentrant circuit can only form in the region directly below the ablation lesion, that is, a circuit can only form if it is at a coordinate corresponding to the $x$ and $y$ range ablated, but with depth greater than that of the ablation lesion. The simulation is now restarted for 10000 time steps and we observe whether or not a reentrant circuit is detected. If yes, the local ablation has failed. If no, the local ablation has been successful. This process is repeated for a range of ablation depths $z$ from a single node layer to the full tissue depth $z=25$, using identical tissues such that different depth results can be compared like for like.

There are three reasons local ablation might fail: (1) the ablation lesion is not deep enough to destroy a reentrant circuit, (2) the targeted reentrant circuit is destroyed but a different independent circuit is present below the ablation lesion, or (3) the ablation lesion itself decouples nodes such that a circuit which was not present previously can now form by anchoring to the ablation lesion itself. 


\section{APPENDIX D: FURTHER MODEL RESULTS}

\section{Activation maps}

In the model, we observe a wide variety of surface activation maps. These can be characterized as full surface reentry (where the active wavefront is visible for the full reentry cycle), partial surface reentry (where only part of the reentry cycle is visible), single location breakthrough points, and multiple quasisimultaneous breakthrough points. The surface activity that is observed in any given tissue depends on the depth of the reentrant circuit as well as the level of connectivity in the tissue. Close-up breakthrough point activation maps are shown in Fig. 11; full and partial reentry maps are shown in Fig. 12.

For a reentrant circuit located in the bulk, both the endocardial and epicardial activation maps are similar, as shown in Fig. 13. A stronger delay can be seen for circuits on one surface. Reentrant circuits are seen on the endocardium and epicardium in Figs. 14 and 15, respectively. Breakthrough points are observed on the surface opposite the reentrant circuit.

\section{Depth of reentrant circuits}

We can measure the depth from the endocardium ( $z$ coordinate) at which reentrant circuits form in the homogeneous and inhomogeneous model. Figure 16 (Fig. 17) shows the distribution of reentrant circuit depths for the homogeneous (inhomogeneous) model at various points on the model's phase space, shown in Fig. 3(a) [Fig. 3(b)] in the main paper. Note, reentrant circuits have a spatial extent and are not only present at a single coordinate in the tissue. However, for simplicity, the algorithm to identify reentrant circuits only identifies the breakthrough point of the isolated fiber in a reentrant circuit, corresponding to point 1 in the activation maps shown in Fig. 4 of the main paper.

In the homogeneous model, the fiber structure is statistically equivalent across the whole tissue. Hence, there is no distinction between the endocardial surface at $z=0$ and the epicardial surface at $z=24$. Figure 16 shows that, in paroxysmal AF, reentrant circuits form preferentially near the atrial surfaces. As nodes continue to decouple and the model approaches persistent AF, reentrant circuits form uniformly in the bulk. This dependence of reentrant circuit depth on AF persistence is found to exist for models with strong longitudinal and weak transverse coupling (Fig. 16 left column), equal longitudinal and transverse coupling (Fig. 16 central column), and weak longitudinal and transverse coupling (Fig. 16 right column). However, reentrant circuits cluster around the atrial surfaces more closely for strong longitudinal and weak transverse coupling than for weak longitudinal and strong transverse coupling.

As a result, when the fiber orientation is varied in the inhomogeneous model, reentrant circuits will form preferentially in the regions with the strongest longitudinal coupling. This breaks the symmetry in reentrant circuit distribution between the endocardium and the epicardium in the inhomogeneous model. In the inhomogeneous model for paroxysmal AF, reentrant circuits are found to cluster preferentially at the atrial surfaces, as opposed to uniformly in the bulk, and at the endocardium, as opposed to the epicardium. As AF progresses, the asymmetry between epicardium and endocardium weakens and reentrant circuits move deeper into the bulk of the atrial wall. Hence, the average distance of reentrant circuits from the endocardial surface increases as AF becomes more persistent. As reentrant circuits move deeper into the atrial wall, they become harder to ablate.

\section{Ablation in the model}

We can simulate ablation in the model by permanently disabling a set of nodes corresponding to the desired ablation lesion (see Appendix C). In clinical practice, ablation is carried out by burning lesions from the endocardial surface.

By ablating active reentrant circuits in the model, AF can be terminated and the model will return to sinus rhythm. An example of this process is shown in Fig. 18. By ablating part of the reentrant circuit, the back-propagating excitation is blocked from reentering the excitable tissue and the fibrillatory activity dissipates.

In clinical practice, ablation lesions can typically only penetrate up to $2 \mathrm{~mm}$ into the atrial wall from the endocardial surface. The atria is $1-7 \mathrm{~mm}$ thick [14], averaging approximately $2.5 \mathrm{~mm}$ [55]. As a result, reentrant circuits which are far from the endocardium may be difficult to destroy using ablation. This is shown in Fig. 6 of the main paper.

\section{Robustness of model results}

The results presented in the main paper are for a tissue with specific thickness and a single choice of fiber orientations. Figure 3(b) is for the model with a thickness of 25 nodes and for fiber orientation parameters of $\Delta \theta_{\text {endo }}=24^{\circ}$ at the endocardium $(z=0)$ and $\Delta \theta_{\text {epi }}=42^{\circ}$ at the epicardium $(z=$ $24)$. These values have not been chosen arbitrarily. The tissue thickness corresponds to the average atrial thickness noted in Ref. [55], and the values for the variation in fiber orientation are taken directly from the experimental studies on transmural reentrant circuits $[14,16]$.

The core results presented in the main paper are robust against changes in the thickness of the tissue and against different choices of fiber orientation. To assess the robustness, we have tested the model for different tissue thicknesses and different values of the fiber orientation. Figure 19 shows the risk curves and associated driver depth distributions for the model for thinner (ten nodes, $1.0 \mathrm{~mm}$ ) and thicker tissue (50 nodes, $5.0 \mathrm{~mm}$ ), and for different choices of fiber orientation parameters on the endo- and epicardium.

The fiber orientation parameters tested cover a range of possible cases that might be relevant to the atria. Figures 19(a) and 19(b) correspond to strong longitudinal coupling on the endocardium, with uniform coupling on the epicardium. Figures 19(c) and 19(d) correspond to the moderate longitudinal coupling on the endocardium and almost uniform coupling on the epicardium (these parameters match those used in the main paper). Figures 19(e) and 19(f) correspond to very mild longitudinal coupling on the endocardium and very mild transverse coupling on the epicardium. Finally, Figs. 19(g) and 19(h) correspond to uniform coupling on the endocardium and strong longitudinal coupling on the 

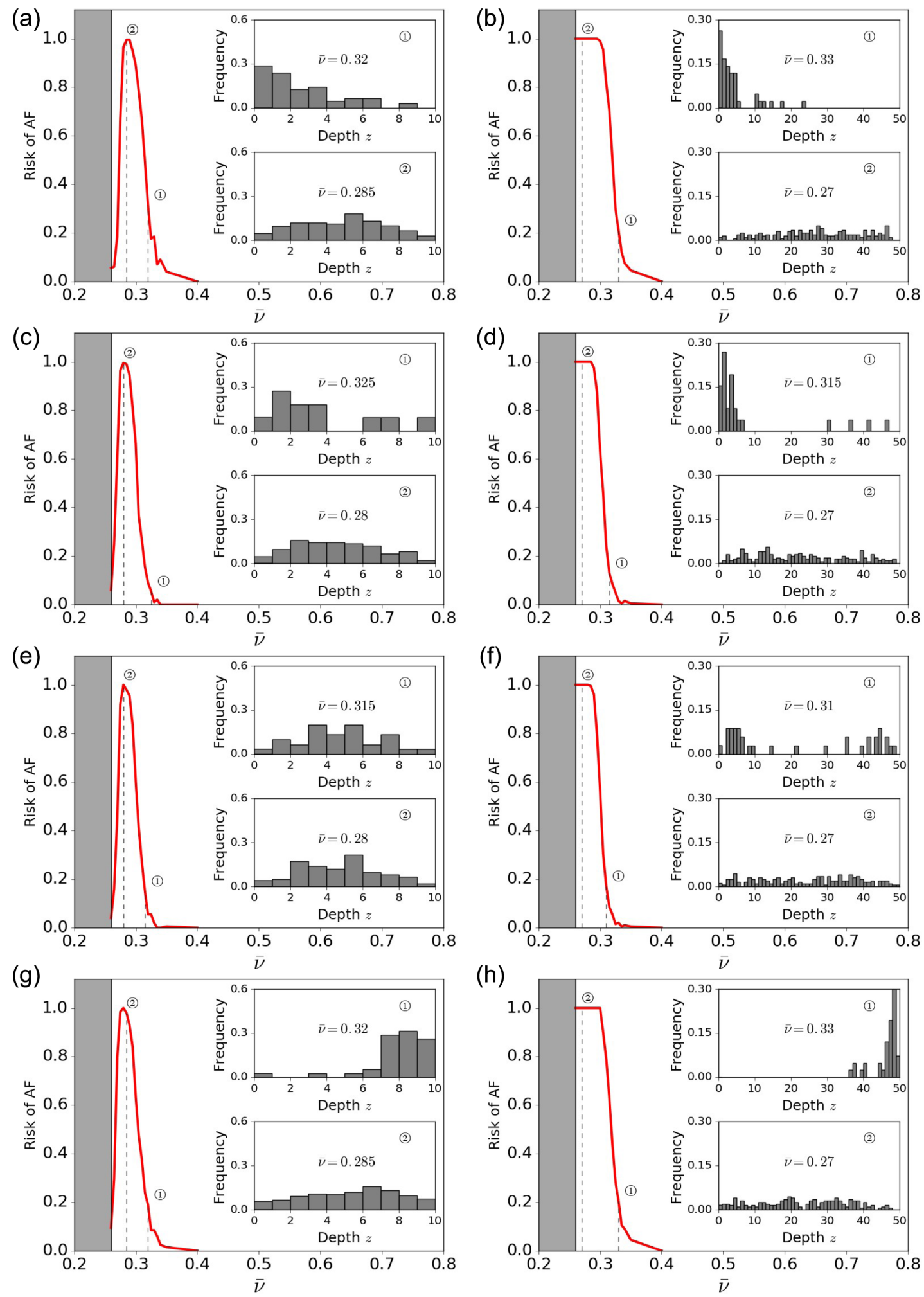

FIG. 19. Inhomogeneous coupling parameter risk curves (red graph) for various choices of fiber orientation and tissue thickness. Inset: Histograms showing the distribution of reentrant circuits driving AF for paroxysmal AF (top) and persistent AF (bottom) as a function of depth $z$ from the endocardium. Left column: Tissue thickness of ten nodes (1.0 mm). Right column: Tissue thickness of $50 \mathrm{nodes}(5.0 \mathrm{~mm})$. (a, b) Strong longitudinal coupling on endocardium, $\Delta \theta_{\text {endo }}=10^{\circ}$, and uniform coupling on the epicardium, $\Delta \theta_{\text {epi }}=45^{\circ}$. (c, d) Moderate longitudinal coupling on endocardium, $\Delta \theta_{\text {endo }}=24^{\circ}$, and nearly uniform coupling on epicardium, $\Delta \theta_{\text {epi }}=42^{\circ}$. Parameters correspond to those used in Fig. 3(b) of the main paper. (e, f) Very mild longitudinal coupling on endocardium, $\Delta \theta_{\text {endo }}=40^{\circ}$, and very mild transverse coupling on epicardium, $\Delta \theta_{\text {epi }}=60^{\circ}$. (g, h) Uniform coupling on endocardium, $\Delta \theta_{\text {endo }}=45^{\circ}$, and strong longitudinal coupling on epicardium, $\Delta \theta_{\text {endo }}=10^{\circ}$. (g) and (h) are identical to (a) and (b) with the endocardium and epicardium swapped. 
epicardium - the reverse case of Figs. 19(a) and 19(b). For models with no change in fiber orientation from endocardium to epicardium, see Fig. 16.

The results consistently show that reentrant circuits are predominantly found near the atrial wall with the strongest longitudinal coupling in the paroxysmal AF case ( $\bar{v}$ is large). However, at lower absolute coupling ( $\bar{v}$ is small), corresponding to the persistent AF case, circuits are distributed evenly between the endo- and epicardium. This result is more pronounced for thicker tissue than thinner tissue, since the spatial extent of circuits allows a circuit to anchor to both the endo- and epicardium if the tissue is too thin. These results indicate that the regions expected to be most at risk in clinical $\mathrm{AF}$ are those with strong longitudinal muscle fibers such as the pectinate muscles identified in Ref. [14]. A detailed discussion of the robustness of the 2D model can be found in Ref. [58].

\section{APPENDIX E: VIDEO}

Video of the activation patterns observed in the model can be found in the Supplemental Material [37]. Captions for the video are listed below. All video are for the inhomogeneous model where the average node-to-node coupling is fixed, $\bar{v}=$ constant, and variation in fiber direction varies linearly from $\Delta \theta_{\text {endo }}=24^{\circ}$ at the endocardium $(z=0)$ to $\Delta \theta_{\text {epi }}=42^{\circ}$ at the epicardium $(z=24)$. The model has dimensions $L_{x}=$ $L_{y}=200$ and $L_{z}=25$ as outlined in the model definition in Appendix A. The remaining model parameters are $\delta=0.05$, $\epsilon=0.05, \tau=50$, and $T=220$.

\section{Videos A}

Video A features a close-up video of the endocardium and the epicardium showing the spontaneous emergence of AF in the inhomogeneous model, $\bar{v}=0.31$. A reentrant circuit forms near the endocardial surface. This video corresponds to Figs. 1 and 4(b) in the main paper. The epicardial breakthrough is shown in Fig. 11(a).

\section{Videos B}

Video B features a close-up video of the endocardium and the epicardium showing the spontaneous emergence of $\mathrm{AF}$ in the inhomogeneous model, $\bar{v}=0.33$. A reentrant circuit forms near the endocardial surface. This video corresponds to Fig. 14 in Appendix D. The epicardial breakthrough is shown in Fig. 11(b).

\section{Videos $\mathrm{C}$}

Video $C$ features a close-up video of the endocardium and the epicardium showing the spontaneous emergence of AF in the inhomogeneous model, $\bar{v}=0.32$. A reentrant circuit forms near the endocardial surface. The epicardial breakthrough is shown in Fig. 11(c).

\section{Videos D}

Video D features a close-up video of the endocardium and the epicardium showing the spontaneous emergence of AF in the inhomogeneous model, $\bar{v}=0.31$, and a cross section through the atrial wall showing the formation of a reentrant circuit near the endocardial surface and the propagation of fibrillatory waves through the atrial wall to the epicardium. The endocardial view in this video corresponds to Fig. 12(b). The cross section corresponds to Fig. 2 in the main paper.

\section{Videos $\mathbf{E}$}

Video E features a close-up video of the endocardium and the epicardium showing the spontaneous emergence of AF in the inhomogeneous model, $\bar{v}=0.32$. A reentrant circuit forms near the endocardial surface. The epicardial breakthrough is shown in Fig. 11(e).

\section{Video F}

Video $\mathrm{F}$ features a full scale video showing the simultaneous activation patterns on the endocardium and epicardium, and cross sections through the atrial wall, $\bar{v}=0.32$. The video shows many of the key features of emergent AF in the model. The model starts in sinus rhythm before a single reentrant circuit forms. This circuit forms temporarily and dies out after one cycle. This temporary circuit forms three times before a new sustained reentrant circuit emerges near the endocardium. The new sustained circuit drives fibrillatory activity across the tissue blocking new wavefronts from entering the tissue at the pacemaker, $x=0$. Shortly after time 2000, the reentrant circuit is ablated and fibrillatory waves dissipate. However, the interaction between the dissipating fibrillatory waves and a new pacemaker wavefront results in a new short lived circuit forming at time 2500. The new fibrillatory waves dissipate before the tissue returns to sinus rhythm. Note that the ablation lesion disrupts all future sinus rhythm wavefronts, although the effect is small.

\section{Video G}

Video $G$ features a full scale video showing the emergence of AF before being terminated by ablation, $\bar{v}=0.33$. The reentrant circuit forms near the endocardium before being destroyed by ablation. The fibrillatory waves dissipate before the tissue returns to sinus rhythm (see Fig. 18).
[1] N. J. Patel, V. Atti, R. D. Mitrani, J. F. Viles-Gonzalez, and J. J. Goldberger, Heart 104, 1989 (2018).

[2] S. Nattel, Nature (London) 415, 219 (2002).

[3] U. Schotten, D. Dobrev, P. G. Platonov, H. Kottkamp, and G. Hindricks, J. Intern. Med. 279, 428 (2016).
[4] V. V. Fedorov and B. J. Hansen, JACC: Clin. Electrophysiol. 4, 84 (2018).

[5] S. Nattel and D. Dobrev, Circ. Res. 120, 1396 (2017).

[6] I. Mann, B. Sandler, N. Linton, and P. Kanagaratnam, Arrhythm. Electrophysiol. Rev. 7, 49 (2018). 
[7] A. Verma, C.-y. Jiang, T. R. Betts, J. Chen, I. Deisenhofer, R. Mantovan, L. Macle, C. A. Morillo, W. Haverkamp, R. Weerasooriya et al., New Engl. J. Med. 372, 1812 (2015).

[8] L. Macle, P. Khairy, R. Weerasooriya, P. Novak, A. Verma, S. Willems, T. Arentz, I. Deisenhofer, G. Veenhuyzen, C. Scavée et al., Lancet 386, 672 (2015).

[9] S. M. Narayan, M. N. Vishwanathan, C. A. B. Kowalewski, T. Baykaner, M. Rodrigo, J. A. B. Zaman, and P. J. Wang, Rev. Port. Cardiol. 36, 9 (2017).

[10] K. Shivkumar, K. A. Ellenbogen, J. D. Hummel, J. M. Miller, and J. S. Steinberg, J. Cardiovasc. Electrophysiol. 23, 1277 (2012).

[11] S. Lee, J. Sahadevan, C. M. Khrestian, I. Cakulev, A. Markowitz, and A. L. Waldo, Circulation 132, 2108 (2015).

[12] S. M. Narayan, D. E. Krummen, P. Clopton, K. Shivkumar, and J. M. Miller, J. Am. Coll. Cardiol. 62, 138 (2013).

[13] M. Haïssaguerre, M. Hocini, A. Denis, A. J. Shah, Y. Komatsu, S. Yamashita, M. Daly, S. Amraoui, S. Zellerhoff, M.-Q. Picat et al., Circulation 130, 530 (2014).

[14] B. J. Hansen, J. Zhao, T. A. Csepe, B. T. Moore, N. Li, L. A. Jayne, A. Kalyanasundaram, P. Lim, A. Bratasz, K. A. Powell et al., Eur. Heart J. 36, 2390 (2015).

[15] B. J. Hansen, T. A. Csepe, J. Zhao, A. J. Ignozzi, J. D. Hummel, and V. V. Fedorov, Circ. Arrhythm. Electrophysiol. 9, e004398 (2016).

[16] T. A. Csepe, B. J. Hansen, and V. V. Fedorov, Trends Cardiovas. Med. 27, 1 (2017).

[17] T. Baykaner, A. J. Rogers, G. L. Meckler, J. A. B. Zaman, R. Navara, M. Rodrigo, M. Alhusseini, C. A. B. Kowalewski, M. N. Viswanathan, S. M. Narayan et al., Circ. Arrhythm. Electrophysiol. 11, e006119 (2018).

[18] J. A. B. Zaman, W. H. Sauer, M. I. Alhusseini, T. Baykaner, R. T. Borne, C. A. B. Kowalewski, S. Busch, P. C. Zei, S. Park, M. N. Viswanathan et al., Circ. Arrhythm. Electrophysiol. 11, e005258 (2018).

[19] S. Nattel, F. Xiong, and M. Aguilar, Nat. Rev. Cardiol. 14, 509 (2017).

[20] H. Calkins, G. Hindricks, R. Cappato, Y.-H. Kim, E. B. Saad, L. Aguinaga, J. G. Akar, V. Badhwar, J. Brugada, J. Camm et al., Heart Rhythm 14, e275 (2017).

[21] C. X. Wong, A. N. Ganesan, and J. B. Selvanayagam, Eur. Heart J. 38, 1294 (2017).

[22] C. H. Roney, C. D. Cantwell, J. D. Bayer, N. A. Qureshi, P. B. Lim, J. H. Tweedy, P. Kanagaratnam, N. S. Peters, E. J. Vigmond, and F. S. Ng, Circ. Arrhythm. Electrophysiol. 10, e004899 (2017).

[23] K. Christensen, K. A. Manani, and N. S. Peters, Phys. Rev. Lett. 114, 028104 (2015).

[24] R. H. Clayton, O. Bernus, E. M. Cherry, H. Dierckx, F. H. Fenton, L. Mirabella, A. V. Panfilov, F. B. Sachse, G. Seemann, and H. Zhang, Prog. Biophys. Mol. Bio. 104, 22 (2011).

[25] N. Wiener and A. Rosenblueth, Arch. Inst. Cardiol. Mex. 16, 205 (1946).

[26] G. Moe, Am. Heart. J. 58, 59 (1959).

[27] V. Krinsky, Cybernetics Problems 20, 59 (1968).

[28] S. Alonso and M. Bär, Phys. Rev. Lett. 110, 158101 (2013).
[29] E. Vigmond, A. Pashaei, S. Amraoui, H. Cochet, and M. Haïssaguerre, Heart Rhythm 13, 1536 (2016).

[30] S. Alonso, R. W. dos Santos, and M. Bär, PloS One 11, e0166972 (2016).

[31] Y. T. Lin, E. T. Y. Chang, J. Eatock, T. Galla, and R. H. Clayton, J. Royal Soc. Interface 14, 20160968 (2017).

[32] D. Makowiec, J. Wdowczyk, and Z. R. Struzik, Front. Physiol. 9, 1859 (2019).

[33] T. A. Gokhale, E. Medvescek, and C. S. Henriquez, Chaos 27, 093909 (2017).

[34] S. A. Niederer, J. Lumens, and N. A. Trayanova, Nat. Rev. Cardiol. 16, 100 (2019).

[35] M. S. Spach and J. P. Boineau, Pacing Clin. Electrophysio. 20, 397 (2006).

[36] M. F. McGillivray, W. Cheng, N. S. Peters, and K. Christensen, Roy. Soc. Open Sci. 5, 172434 (2018).

[37] See Supplemental Material at http://link.aps.org/supplemental/ 10.1103/PhysRevE.100.062406 for videos of activation patterns as described in Appendix E.

[38] H. Cochet, R. Dubois, S. Yamashita, N. Al Jefairi, B. Berte, J.-M. Sellal, D. Hooks, A. Frontera, S. Amraoui, A. Zemoura et al., JACC: Clin. Electrophysiol. 4, 17 (2018).

[39] V. Swarup, T. Baykaner, A. Rostamian, J. P. Daubert, J. Hummel, D. E. Krummen, R. Trikha, J. M. Miller, G. F. Tomassoni, and S. M. Narayan, J. Cardiovasc. Electr. 25, 1284 (2014).

[40] R. O. Y. Beinart, S. Abbara, A. Blum, M. Ferencik, K. Heist, J. Ruskin, and M. Mansour, J. Cardiovasc. Electrophysiol. 22, 1232 (2011).

[41] S. Kumar, C. R. Barbhaiya, S. Balindger, R. M. John, L. M. Epstein, B. A. Koplan, U. B. Tedrow, W. G. Stevenson, and G. F. Michaud, J. Atr. Fibrillation 8, 1189 (2015).

[42] K. Christensen and N. R. Moloney, Complexity and Criticality (Imperial College, London, 2005).

[43] A. A. Saberi, Phys. Rep. 578, 1 (2015).

[44] M. Haïssaguerre, P. Jaïs, D. C. Shah, A. Takahashi, M. Hocini, G. Quiniou, S. Garrigue, A. Le Mouroux, P. Le Métayer, and J. Clémenty, New Engl. J. Med. 339, 659 (1998).

[45] S. Yen Ho, D. Sanchez-Quintana, J. A. Cabrera, and R. H. Anderson, J. Cardiovasc. Electrophysiol. 10, 1525 (1999).

[46] C. Weiss, A. Gocht, S. Willems, M. Hoffmann, T. Risius, and T. Meinertz, Pacing Clin. Electrophysio. 25, 1352 (2002).

[47] R. Weerasooriya, P. Jaïs, C. Scavée, L. Macle, D. C. Shah, T. Arentz, J. A. Salerno, F. Raybaud, K.-J. Choi, M. Hocini, J. Clémenty, and M. Haïssaguerre, J. Cardiovasc. Electrophysiol. 14, 1173 (2003).

[48] G. S. Ramlugun, B. Thomas, V. N. Biktashev, D. P. Fraser, I. J. LeGrice, Z. J. Smaill, Bruce H., and I. V. Biktasheva, arXiv:1809.01186 (2018).

[49] M. Falkenberg, D. Hickey, L. Terrill, A. Ciacci, N. S. Peters, and K. Christensen, in Proceedings of the 2019 Computing in Cardiology Conference (IEEE, New York, 2019), Vol. 46, pp. 1-4.

[50] A. Sau, S. Al-Aidarous, J. Howard, J. Shalhoub, A. Sohaib, M. Shun-Shin, P. G. Novak, R. Leather, L. D. Sterns, C. Lane et al., EP Europace 21, 1176 (2019). 
[51] J. Zhao, T. D. Butters, H. Zhang, A. J. Pullan, I. J. LeGrice, G. B. Sands, and B. H. Smaill, Circ. Arrhythm. Electrophysiol. 5, 361 (2012).

[52] Y. Wang, Z. Xiong, A. Nalar, B. J. Hansen, S. Kharche, G. Seemann, A. Loewe, V. V. Fedorov, and J. Zhao, Comput. Biol. Med. 114, 103444 (2019).

[53] R. M. Lang, M. Bierig, R. B. Devereux, F. A. Flachskampf, E. Foster, P. A. Pellikka, M. H. Picard, M. J. Roman, J. Seward, J. S. Shanewise, S. D. Solomon et al., J. Am. Soc. Echocardiogr. 18, 1440 (2005).
[54] A. Maceira, J. Cosín-Sales, M. Roughton, S. Prasad, and D. Pennell, J. Cardiovasc. Magn. Reson. 12, 65 (2010).

[55] K. Nakamura, N. Funabashi, M. Uehara, M. Ueda, T. Murayama, H. Takaoka, and I. Komuro, Int. J. Cardiol. 148, 139 (2011).

[56] R. A. Luke and J. E. Saffitz, J. Clin. Investig. 87, 1594 (1991).

[57] S. Verheule, E. Wilson, T. Everett, S. Shanbhag, C. Golden, and J. Olgin, Circulation 107, 2615 (2003).

[58] K. Manani, Ph.D. thesis, Imperial College London, 2016. 